ESAIM: M2AN 46 (2012) 1081-1106

DOI: $10.1051 / \mathrm{m} 2 \mathrm{an} / 2011078$
ESAIM: Mathematical Modelling and Numerical Analysis

www.esaim-m2an.org

\title{
REDUCED RESISTIVE MHD IN TOKAMAKS WITH GENERAL DENSITY
}

\author{
BRuno Després ${ }^{1}$ AND RÉmy SART ${ }^{2}$
}

\begin{abstract}
The aim of this paper is to derive a general model for reduced viscous and resistive Magnetohydrodynamics (MHD) and to study its mathematical structure. The model is established for arbitrary density profiles in the poloidal section of the toroidal geometry of Tokamaks. The existence of global weak solutions, on the one hand, and the stability of the fundamental mode around initial data, on the other hand, are investigated.
\end{abstract}

Mathematics Subject Classification. 93A30, 35Q35, 76E25, 82D10.

Received December 23, 2010. Revised December 2, 2011.

Published online February 13, 2012.

\section{INTRODUCTION}

Strong magnetic field are used to confine a plasma in Tokamaks, so that the conditions needed for thermonuclear fusion are reachable [3,18]. The stability of magnetic configuration may be studied with full 3D MHD models $[11,32]$. Reduced resistive MHD model may be obtained from a 2D simplification of full 3D MHD models with resistivity following the seminal work [25]: we refer to $[2,37]$ for cylindrical models and to $[8,9]$ for models in toroidal geometry. In all cases the unknowns are some scalar potentials which are defined in a cut of the initial 3D domain: the cut is planar in $[2,37]$ and it is a poloidal section of a torus in $[8,9]$. Other generalized reduced MHD models may be found in [26].

The first aim of this work is to derive an original and more general reduced resistive MHD model. With respect to the usual reduced resistivity models $[2,8,9,34,37,40,41]$, we need less severe assumptions on the density profile, as it is explained in Figure 2. In our work the density profile is a general given function. To our understanding all previous models are special cases of our model. In $[2,37]$ the density is constant and this means that the flow is assumed to be incompressible. In $[8,9]$ the density is scaled as $R^{-2}$ and it corresponds to a flow in rotation and in inertial equilibrium (see Rem. 2.5). Traditionally [8,9], the family of $2 \mathrm{D}$ reduced MHD models is derived using an assumption of small curvature $(\varepsilon \ll 1)$ and an assumption of small ratio of fluid pressure over magnetic pressure $(\beta \ll 1)$. For the ITER project the curvature is moderate $(\varepsilon \approx 0.3)$, so it is better to derive the model without using expansion with respect to $\varepsilon$ : this is precisely what we do in Section 3 , even if it is possible to recover the basic reduced MHD model as a limit of our model (see Rem. 2.4). Since the fluid pressure does not show up in the final model, it means that we implicitly assume a small $\beta$ regime. In consequence the balance of the material pressure and the magnetic pressure is not studied directly with the

\footnotetext{
Keywords and phrases. Tokamaks, reduced Magnetohydrodynamics.

1 Laboratoire Jacques-Louis Lions, Université Paris VI, 4 place Jussieu, 75015 Paris, France. despres@ann.jussieu.fr

2 École Supérieure d'Ingénieurs Léonard de Vinci, 92916 Paris-La Défense, France. remy.sart@devinci.fr
} 


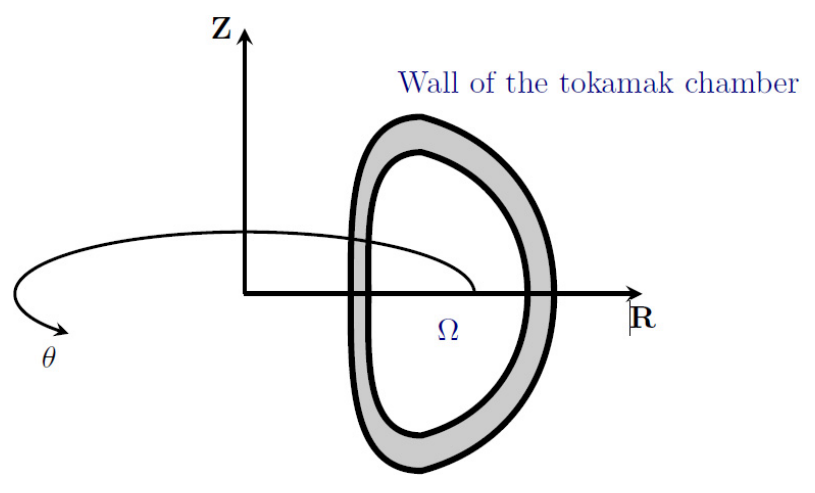

FigurE 1. Schematic description of the poloidal section of a tokamak. The poloidal section is represented by the grey region plus the white region $\Omega$. The main part of the plasma is assumed to be in the white region. The models developed in this work are defined in the $2 \mathrm{D}$ domain $\Omega$.

family of model considered in this work. If one is interest by such phenomenon it is much better to formulate the problem as a free boundary problem (some references to be found in $[5-7,10,15,19,38]$ and therein). Reduced resistive magnetohydrodynamics models have recently been used [13] to study the Current Hole, which is a special type of instability appearing in Tokamaks. Essentially one observes that a stationary physical current profile becomes unstable away from the boundary in the core of the domain and is replaced by a profile with almost zero amplitude. The "Current Hole" phenomena has been indirectly observed in JET [23] and JT-60 [21] and is a scenario for the ITER machine. It is of major physical interest to better understand the Current Hole in view of the ITER project, see [20] and references therein. Following [13] we consider that reduced MHD models can be helpful in this direction. The boundary conditions that we use have very little influence on the Current Hole simulations reported in $[13,14]$. In our work we use a mixed of Dirichlet and Neumann conditions $(2.7)$, but it can be replaced with no harm with pure Dirichlet boundary conditions (2.8). Even it is far to be the case in Tokamaks, we will consider that the exterior boundary is smooth because it fits with the Current Hole simulations of $[13,14]$.

The second aim is to study some mathematical properties of this general model: in this work we focus on the existence of weak solutions in order to establish a mathematical foundation for the simulations reported in $[13,14]$. The model is endowed with an important energy identity, see (4.1) and further generalization. The stability results of this work are based on this energy estimate. Two types of stability are observed: stability of unsteady weak solutions which yields existence, and stability of particular stationary solutions which are constructed from the first eigenfunction of the Grad-Shafranov operator. An interesting feature of our stability estimates around the first eigenfunction of the Grad-Shafranov operator is that they improve for small $\eta$.

The organization of this work is as follows. In Section 2 we present the notations and the reduced model. Section 3 is devoted to the derivation of the model from the full system viscous resistive MHD. Some formal identities are derived in Section 4. We study the existence of weak solutions in Section 5 . We prove some stability estimates for special profiles in Section 6 .

\section{GeOMETRY And nOtations}

The toroidal geometry of a generic tokamak is depicted in Figure 1. The geometry is a torus obtained by the rotation of a poloidal simply connected section around the axis $Z$. We will use the cylindrical coordinates $(R, \theta, Z)$ which are related to the standard Cartesian coordinates $(X, Y, Z)$ through

$$
\left\{\begin{array}{l}
X=R \cos \theta \\
Y=R \sin \theta
\end{array}\right.
$$




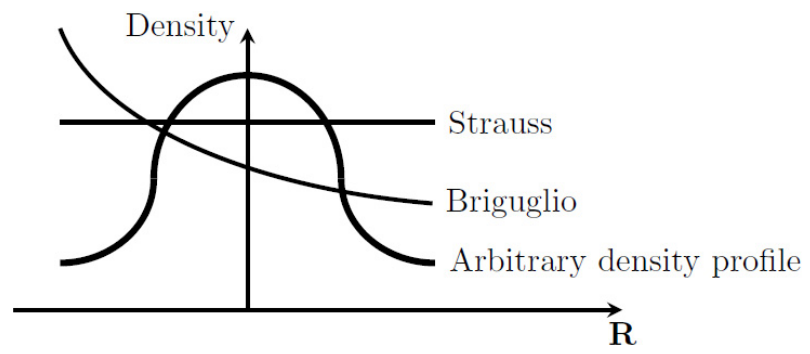

Figure 2. Cut of the density profile inside the Tokamaks chamber $\Omega$. The abscissa is $R \in$ ] $R^{-} R^{+}$[ with $0<R^{-}<R^{+}$. The Strauss profile refers to the incompressible model [2,13,37]; the Briguglio profile refers to the $\rho R^{2}$ constant profile used in $[8,9,13]$; the arbitrary profile corresponds to the new model proposed in this work. We believe that this profile is closer to the real situation in Tokamaks because the density of the plasma is higher in the core of the Tokamak.

We assume that the interesting part of the plasma is confined in a two dimensional poloidal domain $\Omega$ (see Fig. 1). It means that the plasma is confined in the three dimensional domain

$$
(R, Z, \theta) \in \Omega \times[0,2 \pi[.
$$

The $2 \mathrm{D}$ domain $\Omega$ is assumed to have smooth boundary $\partial \Omega$. We will make use of the local direct frame defined by the three unit vectors

$$
\mathbf{e}_{Z}=\nabla Z, \quad \mathbf{e}_{R}=\nabla R, \quad \mathbf{e}_{\theta}=\mathbf{e}_{Z} \wedge \mathbf{e}_{R} .
$$

\subsection{System of viscous resistive MHD equations}

The starting point of the modelling is the full system of viscous resistive MHD equations with source term

$$
\left\{\begin{array}{l}
\partial_{t} \rho+\nabla \cdot(\rho \mathbf{u})=0, \\
\partial_{t} \mathbf{B}=\nabla \wedge(\mathbf{u} \wedge \mathbf{B})-\eta \nabla \wedge(\nabla \wedge \mathbf{B}-\mathbf{F}), \\
\partial_{t}(\rho \mathbf{u})+\nabla \cdot(\rho \mathbf{u} \otimes \mathbf{u})+\nabla p=\mathbf{J} \wedge \mathbf{B}+\nu \Delta \mathbf{u}, \mathbf{J}=\nabla \wedge \mathbf{B} .
\end{array}\right.
$$

In equation (2.1), $\rho$ is the density, $\mathbf{u}$ is the velocity, $\mathbf{B}$ is the magnetic field and $\mathbf{J}$ is the current. The resistivity coefficient $\eta \geq 0$ and the equivalent viscosity coefficient $\nu \geq 0$ are non negative to respect the causality of the equations. In the momentum equation the last term is not the exact viscous operator, but only a simplified one commonly used in plasma physics [13]. The final form of the viscous term will be adapted to the mathematical structure of the reduced model in Remark 3.2. The source term is $\mathbf{F}$. It represents a forcing term that one adds to the induction equation in order to obtain equilibrium. It can be for example an external magnetic field, which even if it may seem unrealistic, is essential to obtain physically relevant MHD numerical simulations $[13,20,30,31]$. This vector is toroidal, that is parallel to $\mathbf{e}_{\theta}$. In some recent numerical developments $[20,31]$ the forcing term represents what is a called a bootstrap current

$$
\mathbf{F}=\mathbf{J}_{\text {boot }}=-J_{\text {boot }} \frac{\mathbf{e}_{\theta}}{R} .
$$

Explanations about the toroidal bootstrap current is to be found in modern neo-classical transport theory of magnetic plasma in Tokamaks, see [18,32]. Neo-classical transport theories are based on generalized Ohm's law, like for the Hall current or the Biermann pressure. In all quoted references $J_{\text {boot }}$ is a function of either the density $\rho$, the pressure $p$ or of a mixed of these quantities. For example Freidberg [18] proposes to retain

$$
J_{\mathrm{boot}} \approx-C \frac{\bar{T}}{F_{0}} \frac{\partial n}{\partial r}
$$


where $n$ is the number of ions (proportional to the density $\rho$ ), $\bar{T}$ the mean value of the temperature, $F_{0}$ is proportional to the the mean value of the magnetic field (2.3) and $C$ is a geometric factor. In this formula $r$ is the radial variable in $\Omega$ (that is $r=0$ at the center of $\Omega$ ). Ultimately it is possible to simplify $J_{\mathrm{boot}} \approx-C \frac{\partial \rho}{\partial r}$. Another completely different approach could be to identify the current: in this direction we refer to [4]. In this work we will consider that the source term $\mathbf{F}=-F \frac{\mathbf{e}_{\theta}}{R}$ is a given smooth function.

\subsection{Reduced resistive model with general density}

The reduced model used in this work is a simplification of (2.1) where one uses usual potential formulas for both the magnetic field and the velocity. The magnetic field is represented as $[11,18,32]$

$$
\mathbf{B}=F_{0} \nabla \theta+\nabla \psi \wedge \nabla \theta .
$$

The $\nabla$ operator is defined in the $X, Y, Z$ system of coordinates. For the simplicity of mathematical developments, the coefficient $F_{0} \neq 0$ is given and constant in space and time. Since $\nabla \theta=\frac{1}{R} \mathbf{e}_{\theta}$ then $\mathbf{B}=\frac{F_{0}}{R} \mathbf{e}_{\theta}+\frac{1}{R} \nabla \psi \wedge \mathbf{e}_{\theta}$ is the sum of a toroidal vector and of a poloidal vector. By definition $\nabla \psi \wedge \nabla \theta=\nabla \wedge(\psi \nabla \theta)$. It is immediate to check that the angular variable $\theta$ is an harmonic function that is $\Delta \theta=0$. Therefore the magnetic field is by construction a free divergence vector $\nabla \cdot \mathbf{B}=0$. In Tokamaks the first contribution $\frac{F_{0}}{R} \mathbf{e}_{\theta}$ is the principal one. The second term is a purely poloidal perturbation $(\nabla \psi \wedge \nabla \theta) \cdot \mathbf{e}_{\theta}=0$.

Flows such that the density is unchanged correspond to $\nabla \cdot(\rho \mathbf{u})=0$. It is therefore convenient to assume that the velocity is represented in a form similar to (2.3). In this work we disregard the parallel or toroidal part of the velocity, that is we use the representation

$$
\mathbf{u}=\frac{1}{\rho} \nabla \Phi \wedge \nabla \theta=\frac{1}{\rho R} \nabla \Phi \wedge \mathbf{e}_{\theta} .
$$

By construction the velocity is poloidal, $\mathbf{u} \cdot \mathbf{e}_{\theta}=0$. The fact that the toroidal component of the velocity is neglected is an important simplification, and can perhaps be justified for special equilibrium situations where the mean toroidal part of the velocity is small. More general reduced models with toroidal velocity are to be found in $[12,13,24]$ : it must be noted that the mathematical structure of the equation of the toroidal component $u_{\|}$is slightly different; in this work we do not pursue in this direction

The potential representation (2.4) implies that $\nabla \cdot(\rho \mathbf{u})=0$ holds true. Therefore one has by construction that

$$
\partial_{t} \rho+\nabla \cdot(\rho \mathbf{u})=0 .
$$

That is the density a given constant function $\rho(R, Z)$ and and is independent of the time variable.

We need some notations to write the reduced model derived from (2.1)-(2.4). The Poisson brackets of two scalar functions is

$$
[a, b]=\partial_{R} a \partial_{Z} b-\partial_{Z} a \partial_{R} b .
$$

The Grad-Shafranov operator is defined by

$$
\Delta^{\star} \psi=R\left(\partial_{Z}\left(\frac{\partial_{Z} \psi}{R}\right)+\partial_{R}\left(\frac{\partial_{R} \psi}{R}\right)\right)=\partial_{R R} \psi+\partial_{Z Z} \pi-\frac{1}{R} \partial_{R} \psi
$$

The diffusion operator $\Delta_{\rho}$ is defined by

$$
\Delta_{\rho} \Phi=\rho R\left(\partial_{Z}\left(\frac{\partial_{Z} \psi}{\rho R}\right)+\partial_{R}\left(\frac{\partial_{R} \psi}{\rho R}\right)\right)=\Delta^{\star} \Phi-\frac{1}{\rho}\left(\partial_{R} \rho \partial_{R} \Phi+\partial_{Z} \rho \partial_{Z} \Phi\right) .
$$

Definition 2.1 (reduced resistivity MHD model with general density). The model is

$$
\left\{\begin{array}{l}
\partial_{t} \psi=\frac{1}{\rho R}[\psi, \Phi]+\eta \Delta^{\star} \psi-\eta F, \\
\partial_{t} \omega=\frac{1}{\rho R}[\omega, \Phi]-2 \frac{1}{(\rho R)^{2}}[\rho R, \Phi] \omega+\rho R\left[\psi, \frac{1}{\rho R^{2}} \Delta^{\star} \psi\right]+\nu \Delta_{\rho} \omega,
\end{array}\right.
$$


with $\omega=\Delta_{\rho} \Phi$ is the reduced vorticity. The domain is $(R, Z) \in \Omega$. The density profile is given and timeindependent. It is assumed to be smooth

$$
\rho \in W^{1, \infty}(\Omega), \quad 0<\rho_{-} \leq \rho \leq \rho_{+} .
$$

The system is supplemented with natural Dirichlet and Neumann boundary conditions

$$
\psi=\Phi=\frac{\partial \Phi}{\partial n}=0 \text { on } \partial \Omega .
$$

The scalar potential $\psi$ is the magnetic flux. The scalar potential $\Phi$ is the velocity potential.

Remark 2.2. The boundary condition (2.7) guarantees that the velocity vanishes at the boundary, that is $\mathbf{u}=0$ on $\partial \Omega$. Other boundary conditions are used in [13]

$$
\psi=\Phi=\omega=0 \text { on } \partial \Omega .
$$

The difference for the simulations reported in [13] is negligible since $\Phi$ is identically zero near the boundary for physical reasons.

Remark 2.3. It is remarkable that the reduced model (2.5) does not account for the principal toroidal part of the magnetic field. This is due to a strong decoupling of the equations between the poloidal part and the toroidal part.

Remark 2.4 (planar geometry with incompressibility). Let us set $\rho=1$ and neglect the effect of curvature in (2.5), that is we set $R=1$ everywhere. The model corresponds to the seminal reduced resistive incompressible model [37] in a cylinder.

Remark 2.5 (cylindrical geometry with a constant $\rho R^{2}$ ). It is described in [13,35]. In our case it is sufficient to set

$$
\rho=\frac{K}{R^{2}}
$$

in the general model (2.5). It corresponds to a situation [33] where the plasma in the torus is in uniform rotation with a constant angular velocity $\omega_{c}$. In this case the centrifugal acceleration is $\rho v^{2}=\rho R^{2} \omega_{c}^{2}$. If this centrifugal force is constant, then the plasma is in some kind of mechanical equilibrium. It corresponds precisely to (2.9).

\section{Derivation of the MODEL}

This section is devoted to the derivation of (2.5) from (2.1) after convenient simplifications.

\subsection{The magnetic equation}

Consider the magnetic equation of $(2.1)$

$$
\partial_{t} \mathbf{B}=\nabla \wedge(\mathbf{u} \wedge \mathbf{B})-\eta \nabla \wedge(\nabla \wedge \mathbf{B}-\mathbf{F})
$$

with the magnetic field represented by (2.3). Since $\mathbf{B}$ is a rotational and $F_{0}$ is constant in time, we get

$$
\partial_{t} \psi \nabla \theta=\mathbf{u} \wedge \mathbf{B}-\eta \nabla \wedge \mathbf{B}+\eta \mathbf{F}+\nabla V
$$

where $\nabla V$ is an unknown gradient for which a gauge condition will be prescribed below. We assume that the potentials are independent of the angle variables, that is

$$
\psi=\psi(R, Z) \text { and } \Phi=\Phi(R, Z) .
$$


Then

$$
\mathbf{u}=\frac{1}{\rho R} \nabla \Phi \wedge \mathbf{e}_{\theta}=\frac{1}{\rho R}\left(-\partial_{Z} \Phi \mathbf{e}_{R}+\partial_{R} \Phi \mathbf{e}_{Z}\right)
$$

and similarly

$$
\mathbf{B}=\frac{F_{0}}{R} \mathbf{e}_{\theta}+\frac{1}{R}\left(-\partial_{Z} \psi \mathbf{e}_{R}+\partial_{R} \psi \mathbf{e}_{Z}\right)
$$

so that

$$
\mathbf{u} \wedge \mathbf{B}=-\frac{F_{0}}{\rho R^{2}}\left(\partial_{Z} \Phi \mathbf{e}_{Z}+\partial_{R} \Phi \mathbf{e}_{R}\right)+\frac{1}{\rho R^{2}}[\psi, \Phi] \mathbf{e}_{\theta} .
$$

To compute $\mathbf{J}=\nabla \wedge \mathbf{B}$ we notice that $\nabla \wedge \mathbf{e}_{R}=\nabla \wedge(\nabla R)=0$, that $\nabla \wedge \mathbf{e}_{Z}=0$ and that $\nabla \wedge \frac{\mathbf{e}_{\theta}}{R}=\nabla \wedge \nabla \theta=0$. So

$$
\mathbf{J}=-\nabla\left(\frac{\partial_{Z} \psi}{R}\right) \wedge \mathbf{e}_{R}+\nabla\left(\frac{\partial_{R} \psi}{R}\right) \wedge \mathbf{e}_{Z}
$$

The first term is

$$
\begin{aligned}
\nabla\left(\frac{\partial_{Z} \psi}{R}\right) \wedge \mathbf{e}_{R} & =\left(\partial_{R}\left(\frac{\partial_{Z} \psi}{R}\right) \mathbf{e}_{R}+\partial_{Z}\left(\frac{\partial_{Z} \psi}{R}\right) \mathbf{e}_{Z}+\frac{1}{R} \partial_{\theta}\left(\frac{\partial_{Z} \psi}{R}\right) \mathbf{e}_{\theta}\right) \wedge \mathbf{e}_{R} \\
& =\partial_{Z}\left(\frac{\partial_{Z} \psi}{R}\right) \mathbf{e}_{\theta} .
\end{aligned}
$$

The second term is

$$
\begin{aligned}
\nabla\left(\frac{\partial_{R} \psi}{R}\right) \wedge \mathbf{e}_{Z} & =\left(\partial_{R}\left(\frac{\partial_{R} \psi}{R}\right) \mathbf{e}_{R}+\partial_{Z}\left(\frac{\partial_{R} \psi}{R}\right) \mathbf{e}_{Z}+\frac{1}{R} \partial_{\theta}\left(\frac{\partial_{R} \psi}{R}\right) \mathbf{e}_{\theta}\right) \wedge \mathbf{e}_{Z} \\
& =-\partial_{R}\left(\frac{\partial_{R} \psi}{R}\right) \mathbf{e}_{\theta} .
\end{aligned}
$$

So

$$
\mathbf{J}=-\left(\partial_{Z}\left(\frac{\partial_{Z} \psi}{R}\right)+\partial_{R}\left(\frac{\partial_{R} \psi}{R}\right)\right) \mathbf{e}_{\theta}=-\frac{1}{R} \Delta^{\star} \psi \mathbf{e}_{\theta} .
$$

Therefore (2.2)-(3.1) reduce to

$$
\partial_{t} \psi \frac{\mathbf{e}_{\theta}}{R}=-\frac{F_{0}}{\rho R^{2}}\left(\partial_{Z} \Phi \mathbf{e}_{Z}+\partial_{R} \Phi \mathbf{e}_{R}\right)+\frac{1}{\rho R^{2}}[\psi, \Phi] \mathbf{e}_{\theta}+\eta \frac{1}{R} \Delta^{\star} \psi \mathbf{e}_{\theta}-\eta F \frac{\mathbf{e}_{\theta}}{R}+\nabla V .
$$

Next we make the usual assumption that $V=V(R, Z)$ is poloidal. So $\nabla V$ is a poloidal vector, that is it has zero component along the toroidal direction $\mathbf{e}_{\theta}$. Taking the scalar product of this equation with $\mathbf{e}_{\theta}$, we obtain the scalar equation

$$
\partial_{t} \psi=\frac{1}{\rho R}[\psi, \Phi]+\eta \Delta^{\star} \psi-\eta F
$$

which is the first ingredient in the reduced model (2.5).

Remark 3.1. One notices that the unknown gradient implicitly satisfies the equilibrium equation

$$
-\frac{F_{0}}{\rho R^{2}}\left(\partial_{Z} \Phi \mathbf{e}_{Z}+\partial_{R} \Phi \mathbf{e}_{R}\right)+\nabla V=0 .
$$

Assuming that $\rho R^{2}=K$ is constant as in (2.9), a solution is $V=-\frac{F_{0}}{K} \Phi$. 


\subsection{The momentum equation}

We start from the non viscous momentum equation

$$
\partial_{t} \mathbf{u}+\mathbf{u} \cdot \nabla \mathbf{u}+\frac{1}{\rho} \nabla p=\frac{\mathbf{J} \wedge \mathbf{B}}{\rho}, \quad \mathbf{J}=\nabla \wedge \mathbf{B} .
$$

Here the viscosity is eliminated for the sake of simplicity. It will be reintroduced at the end of this section. Define the vectorial vorticity

$$
\Omega=\nabla \wedge \mathbf{u}
$$

with the equation

$$
\partial_{t} \Omega+\nabla \wedge(\mathbf{u} \cdot \nabla \mathbf{u})=\nabla \wedge\left(\frac{\mathbf{J} \wedge \mathbf{B}}{\rho}\right)
$$

where we have assume either that $\nabla p$ is small with respect to all other terms (it is a low $\beta$ assumption) or that the pressure is a function of the density so that $\frac{1}{\rho} \nabla p=\nabla q(\rho)$ has zero vorticity. One has

$$
\begin{aligned}
\Omega & =\nabla \wedge\left(\frac{1}{\rho} \nabla \Phi \wedge \nabla \theta\right) \\
& =\frac{1}{\rho} \nabla \wedge(\nabla \Phi \wedge \nabla \theta)-\frac{1}{\rho^{2}} \nabla \rho \wedge(\nabla \Phi \wedge \nabla \theta) \\
& =\frac{1}{\rho}\left(-\frac{1}{R} \Delta^{\star} \Phi \mathbf{e}_{\theta}\right)-\frac{1}{\rho^{2}}((\nabla \rho \cdot \nabla \theta) \nabla \Phi-(\nabla \rho \cdot \nabla \Phi) \nabla \theta) \\
& =\left(-\frac{1}{\rho R} \Delta^{\star} \Phi+\frac{1}{\rho^{2} R}(\nabla \rho \cdot \nabla \Phi)\right) \mathbf{e}_{\theta} .
\end{aligned}
$$

The equation becomes

$$
\Omega=-\frac{1}{\rho R} \Delta_{\rho} \Phi \mathbf{e}_{\theta}
$$

Notice that by construction

$$
\Delta_{\rho} \Phi=\rho R\left(\partial_{R}\left(\frac{1}{\rho R} \partial_{R} \Phi\right)+\partial_{Z}\left(\frac{1}{\rho R} \partial_{Z} \Phi\right)\right)
$$

and

$$
\Delta^{\star}=\Delta_{\rho \equiv 1} .
$$

Next, since $\left(\nabla \psi \cdot \mathbf{e}_{\theta}\right)=0$, the computation of the right hand side $\nabla \wedge\left(\frac{\mathbf{J} \wedge \mathbf{B}}{\rho}\right)$ gives

$$
\frac{\mathbf{J} \wedge \mathbf{B}}{\rho}=-\left(\frac{\Delta^{\star} \psi \mathbf{e}_{\theta}}{\rho R}\right) \wedge\left(F_{0} \frac{\mathbf{e}_{\theta}}{R}+\frac{\nabla \psi \wedge \mathbf{e}_{\theta}}{R}\right)=-\frac{1}{\rho R^{2}} \Delta^{\star} \psi \nabla \psi
$$

We notice that the dominant part $F_{0} \frac{\mathbf{e}_{\theta}}{R}$ of the magnetic field completely vanishes in the calculation of the magnetic pressure $\mathbf{J} \wedge \mathbf{B}$. Therefore

$$
\frac{\mathbf{J} \wedge \mathbf{B}}{\rho}=-\left(\frac{\Delta^{\star} \psi \partial_{R} \psi}{\rho R^{2}} \mathbf{e}_{R}+\frac{\Delta^{\star} \psi \partial_{Z} \psi}{\rho R^{2}} \mathbf{e}_{Z}\right)
$$

and then

$$
\begin{aligned}
\nabla \wedge\left(\frac{\mathbf{J} \wedge \mathbf{B}}{\rho}\right) & =-\left(\nabla\left(\frac{\Delta^{\star} \psi \partial_{R} \psi}{\rho R^{2}}\right) \wedge \mathbf{e}_{R}+\nabla\left(\frac{\Delta^{\star} \psi \partial_{Z} \psi}{\rho R^{2}}\right) \wedge \mathbf{e}_{Z}\right) \\
& =-\left(\partial_{Z}\left(\frac{\Delta^{\star} \psi \partial_{R} \psi}{\rho R^{2}}\right)-\partial_{R}\left(\frac{\Delta^{\star} \psi \partial_{Z} \psi}{\rho R^{2}}\right)\right) \mathbf{e}_{\theta} \\
& =-\left[\psi, \frac{1}{\rho R^{2}} \Delta^{\star} \psi\right] \mathbf{e}_{\theta} .
\end{aligned}
$$


Finally we analyze $\nabla \wedge(\mathbf{u} \cdot \nabla \mathbf{u})$. One has

$$
\mathbf{u}=-\frac{1}{\rho R} \partial_{Z} \Phi \mathbf{e}_{R}+\frac{1}{\rho R} \partial_{R} \Phi \mathbf{e}_{Z}=\alpha \mathbf{e}_{R}+\beta \mathbf{e}_{Z}
$$

where we have set $\alpha=-\frac{1}{\rho R} \partial_{Z} \Phi$ and $\beta=\frac{1}{\rho R} \partial_{R} \Phi$. Therefore

$$
\nabla \mathbf{u}=\alpha \nabla \mathbf{e}_{R}+\mathbf{e}_{R} \otimes \nabla \alpha+\mathbf{e}_{Z} \otimes \nabla \beta .
$$

Noticing that $\nabla \mathbf{e}_{R}=\frac{1}{R} \mathbf{e}_{\theta} \otimes \mathbf{e}_{\theta}$, one obtains

$$
\mathbf{u} \cdot \nabla \mathbf{u}=\left(\alpha \partial_{R} \beta+\beta \partial_{Z} \beta\right) \mathbf{e}_{R}+\left(\alpha \partial_{R} \alpha+\beta \partial_{Z} \alpha\right) \mathbf{e}_{Z}
$$

and

$$
\begin{aligned}
\nabla \wedge(\mathbf{u} \cdot \nabla \mathbf{u}) & =\nabla\left(\alpha \partial_{R} \beta+\beta \partial_{Z} \beta\right) \wedge \mathbf{e}_{R}+\nabla\left(\alpha \partial_{R} \alpha+\beta \partial_{Z} \alpha\right) \wedge \mathbf{e}_{Z} \\
& =\left(\partial_{Z}\left(\alpha \partial_{R} \beta+\beta \partial_{Z} \beta\right)-\partial_{R}\left(\alpha \partial_{R} \alpha+\beta \partial_{Z} \alpha\right)\right) \mathbf{e}_{\theta} \\
& =A \mathbf{e}_{\theta} .
\end{aligned}
$$

One has the identity $A=\alpha \partial_{R}\left(\partial_{Z} \alpha-\partial_{R} \beta\right)+\beta \partial_{Z}\left(\partial_{Z} \alpha-\partial_{R} \beta\right)+\left(\partial_{R} \alpha+\partial_{Z} \beta\right)\left(\partial_{Z} \alpha-\partial_{R} \beta\right)$. By definition

$$
\partial_{Z} \alpha-\partial_{R} \beta=-\partial_{R}\left(\frac{1}{\rho R} \partial_{R} \Phi\right)-\partial_{Z}\left(\frac{1}{\rho R} \partial_{Z} \Phi\right)=-\frac{1}{\rho R} \Delta_{\rho} \Phi
$$

One also has

$$
\begin{aligned}
\partial_{R} \alpha+\partial_{Z} \beta & =-\partial_{R}\left(\frac{1}{\rho R} \partial_{Z} \Phi \mathbf{e}_{R}\right)+\partial_{Z}\left(\frac{1}{\rho R} \partial_{R} \Phi \mathbf{e}_{R}\right) \\
& =\frac{\partial_{R}(\rho R)}{(\rho R)^{2}} \partial_{Z} \Phi-\frac{\partial_{Z}(\rho R)}{(\rho R)^{2}} \partial_{R} \Phi \\
& =\frac{1}{(\rho R)^{2}}[\rho R, \Phi] .
\end{aligned}
$$

Therefore

$$
\begin{aligned}
A & =\frac{\partial_{Z} \Phi}{\rho R} \partial_{R}\left(\frac{\Delta_{\rho} \Phi}{\rho R}\right)-\frac{\partial_{R} \Phi}{\rho R} \partial_{Z}\left(\frac{\Delta_{\rho} \Phi}{\rho R}\right)-\frac{1}{(\rho R)^{3}}[\rho R, \Phi] \\
& =\frac{1}{(\rho R)^{2}}\left[\Delta_{\rho} \Phi, \Phi\right]+2 \frac{1}{(\rho R)^{3}}[\rho R, \Phi] \Delta_{\rho} \Phi .
\end{aligned}
$$

For convenience we define

$$
\omega=\Delta_{\rho} \Phi
$$

This is the usual vorticity if $\rho R$ is constant. By analogy we may call it the vorticity even if $\rho R$ is not a constant. The equation may be written as

$$
-\frac{1}{\rho R} \partial_{t} \omega \mathbf{e}_{\theta}+\frac{1}{(\rho R)^{2}}[\omega, \Phi] \mathbf{e}_{\theta}+2 \frac{1}{(\rho R)^{3}}[\rho R, \Phi] \omega \mathbf{e}_{\theta}=-\left[\psi, \frac{1}{\rho R^{2}} \Delta^{\star} \psi\right] \mathbf{e}_{\theta} .
$$

We finally deduce the vorticity equation with zero viscosity

$$
\partial_{t} \omega=\frac{1}{\rho R}[\omega, \Phi]-2 \frac{1}{(\rho R)^{2}}[\rho R, \Phi] \omega+\rho R\left[\psi, \frac{1}{\rho R^{2}} \Delta^{\star} \psi\right] .
$$

The reduced non viscous resistive MHD model corresponds to (3.2) and (3.3) (but without the $\nu \Delta_{\rho} \Phi$ term). 
Remark 3.2 (discussion of the viscous term in the last equation of (2.5)). The final model (2.5) contains the viscous operator $\nu \Delta_{\rho} \Phi$. In this work we do not derive the reduced viscous operator from the initial operator $\nu \Delta \mathbf{u}$ with exact algebra. Moreover this of course depends on the specific form of the viscous operator which may change as well. We refer to [22] for a similar discussion in the context of MHD models for metal forging. Another difficulty is that the calculations are quite tricky in our context.

This is why we propose to retain the final form of the viscous operator by compatibility with the fundamental energy identity (4.1). Using this design principle $\nu \Delta_{\rho} \Phi$ is the optimal viscous operator that we retain in our work.

\section{IDENTITIES}

We quote several formal identities which are true for regular solutions of the preceding system (2.5)-(2.7).

\subsection{Preservation of the total magnetic flux}

Lemma 4.1. Assume $\eta=\nu=0$. Then regular solutions of (2.5)-(2.7) satisfy

$$
\frac{\mathrm{d}}{\mathrm{dt}} \int_{\Omega} \rho R \psi \mathrm{d} R \mathrm{~d} Z=0
$$

Proof. It comes from

$$
\frac{\mathrm{d}}{\mathrm{dt}} \int_{\Omega} \rho R \psi=\int_{\Omega}[\psi, \Phi]=\int_{\Omega}\left(\partial_{R}\left(\psi \partial_{Z} \Phi\right)-\partial_{Z}\left(\psi \partial_{R} \Phi\right)\right)=\int_{\partial \Omega} \psi \partial_{\tan } \Phi \mathrm{d} \sigma=0 .
$$

In this formula $\partial_{\tan }$ is the tangential derivative. The boundary integral vanishes thanks to the boundary condition (2.7).

\subsection{Preservation of the cross-helicity}

Lemma 4.2. Assume $\eta=\nu=0$. Then regular solutions of (2.5)-(2.7) satisfy

$$
\frac{\mathrm{d}}{\mathrm{dt}} \int_{\Omega} \frac{1}{\rho R} \psi \omega \mathrm{d} R \mathrm{~d} Z=0
$$

Proof. It comes from

$$
\begin{aligned}
\frac{\mathrm{d}}{\mathrm{dt}} \int_{\Omega} \frac{1}{\rho R} \psi \omega & =\int_{\Omega} \frac{1}{\rho R}\left(\omega \partial_{t} \psi+\psi \partial_{t} \omega\right) \\
& =\int_{\Omega} \frac{1}{(\rho R)^{2}} \omega[\psi, \Phi]+\int_{\Omega} \psi\left(\frac{1}{(\rho R)^{2}}[\omega, \Phi]+\left[\frac{1}{(\rho R)^{2}}, \Phi\right] \omega\right)+\int_{\Omega} \psi\left[\psi, \frac{1}{\rho R^{2}} \Delta^{\star} \psi\right] \\
& =\underbrace{\int_{\Omega} \frac{1}{(\rho R)^{2}}[\omega \psi, \Phi]+\int_{\Omega}\left[\frac{1}{(\rho R)^{2}}, \Phi\right] \omega \psi}_{=\int_{\Omega}\left[\frac{\omega \psi}{(\rho R)^{2}}, \Phi\right]}+\int_{\Omega}\left[\frac{\psi^{2}}{2}, \frac{1}{\rho R^{2}} \Delta^{\star} \psi\right] \\
& =0
\end{aligned}
$$

after integration and use of the boundary condition (2.7). 


\subsection{The energy identity}

This energy identity will have fundamental consequences in the sequel.

Proposition 4.3. Assume $\eta \geq 0$ and $\nu \geq 0$. Then regular solutions of (2.5)-(2.7) satisfy

$$
\frac{1}{2} \frac{\mathrm{d}}{\mathrm{dt}} \int_{\Omega}\left(\frac{|\nabla \psi|^{2}}{R}+\frac{|\nabla \Phi|^{2}}{\rho R}\right)+\eta \int_{\Omega} \frac{\left|\Delta^{\star} \psi\right|^{2}}{R}+\nu \int_{\Omega} \frac{\left|\Delta_{\rho} \Phi\right|^{2}}{\rho R}=\eta \int_{\Omega} \frac{F \Delta^{\star} \psi}{R} .
$$

Remark 4.4. This identity controls the total energy of the system which is composed of the magnetic energy (2.3) and of the kinetic energy (2.4).

Proof.

- By multiplying the first equation of $(2.5)$ by $\frac{\Delta^{\star} \psi}{R}$, we get

$$
\int_{\Omega} \partial_{t} \psi \frac{\Delta^{\star} \psi}{R}=\int_{\Omega} \frac{1}{\rho R^{2}}[\psi, \Phi] \Delta^{\star} \psi+\eta \int_{\Omega} \frac{\left|\Delta^{\star} \psi\right|^{2}}{R}-\eta \int_{\Omega} \frac{F \Delta^{\star} \psi}{R}
$$

Integrating by parts and using properties of the Poisson brackets, we obtain

$$
-\int_{\Omega} \partial_{t} \nabla \psi \cdot\left(\frac{\nabla \psi}{R}\right)=-\int_{\Omega}\left[\psi, \frac{1}{\rho R^{2}} \Delta^{\star} \psi\right] \Phi+\eta \int_{\Omega} \frac{\left|\Delta^{\star} \psi\right|^{2}}{R}-\eta \int_{\Omega} \frac{F \Delta^{\star} \psi}{R}
$$

and then

$$
-\frac{1}{2} \frac{\mathrm{d}}{\mathrm{dt}} \int_{\Omega} \frac{|\nabla \psi|^{2}}{R}=-\int_{\Omega}\left[\psi, \frac{1}{\rho R^{2}} \Delta^{\star} \psi\right] \Phi+\eta \int_{\Omega} \frac{\left|\Delta^{\star} \psi\right|^{2}}{R}-\eta \int_{\Omega} \frac{F \Delta^{\star} \psi}{R}
$$

- By multiplying the second equation of $(2.5)$ by $\frac{\Phi}{\rho R}$, we get

$$
\int_{\Omega} \partial_{t} \omega \frac{\Phi}{\rho R}=\int_{\Omega} \frac{1}{(\rho R)^{2}}[\omega, \Phi] \Phi-\int_{\Omega} \frac{2}{(\rho R)^{3}}[\rho R, \Phi] \omega \Phi+\int_{\Omega}\left[\psi, \frac{1}{\rho R^{2}} \Delta^{\star} \psi\right] \Phi+\nu \int_{\Omega} \Delta_{\rho} \omega \frac{\Phi}{\rho R}
$$

By similar calculations, we successively get

$$
-\int_{\Omega} \partial_{t}\left(\frac{\nabla \Phi}{\rho R}\right) \nabla \Phi=\int_{\Omega}\left[\Phi, \frac{1}{(\rho R)^{2}} \Phi\right] \omega-\int_{\Omega} \frac{2}{(\rho R)^{3}}[\rho R, \Phi] \omega \Phi+\int_{\Omega}\left[\psi, \frac{1}{\rho R^{2}} \Delta^{\star} \psi\right] \Phi-\nu \int_{\Omega} \nabla \omega \cdot \frac{\nabla \Phi}{\rho R}
$$

and

$$
\begin{aligned}
-\frac{1}{2} \frac{\mathrm{d}}{\mathrm{dt}} \int_{\Omega} \frac{|\nabla \Phi|^{2}}{\rho R}= & \overbrace{\int_{\Omega}\left[\Phi, \frac{1}{(\rho R)^{2}}\right] \Phi \omega-\int_{\Omega} \frac{2}{(\rho R)^{3}}[\rho R, \Phi] \omega \Phi}^{=0}+\int_{\Omega} \underbrace{[\Phi, \Phi]}_{=0} \frac{1}{(\rho R)^{2}} \omega \\
& +\int_{\Omega}\left[\psi, \frac{1}{\rho R^{2}} \Delta^{\star} \psi\right] \Phi+\nu \int_{\Omega} \omega \frac{\Delta_{\rho} \Phi}{\rho R} \\
= & \int_{\Omega}\left[\psi, \frac{1}{\rho R^{2}} \Delta^{\star} \psi\right] \Phi+\nu \int_{\Omega} \frac{\left|\Delta_{\rho} \Phi\right|^{2}}{\rho R} .
\end{aligned}
$$

- We conclude by summing (4.2) and (4.3). 


\section{Existence OF WEAK SOLUTiOnS}

In this section the existence of weak solutions is investigated. Our goal is to assess that variable density profiles are compatible with the standard theory of such systems for which we refer the reader to the seminal contributions $[27,39]$. General tools for the construction of approximate solutions has already been described. For instance, Schauder fixed point arguments for linearized models has been precisely explained in [28, 29], we also refer to [22] for a modern presentation of the theory in the context of liquid metals. Existence results for approximate models with regularizing extra terms has also been clarified in [17]. Anyway, the convergence of regular approximate functions to weak solutions is the crucial point. Essentially it amounts to showing that the a priori estimates (5.1) controls the continuity of the non linear terms of the general model. The method of construction of the sequence of regular solutions is based on a particular splitting in time of the equation, with the same structure has in the seminal Temam's work [39] for Navier-Stokes equations and related problems. It must be noticed that practical simulations in the context of the numerical simulation of the Current Hole [13,20,21] have been performed with Finite Elements Methods coupled with this spitting strategy [14]: they have indeed shown unconditional stability.

We will assume in this section that $\nu>0, \eta>0$, that (2.6) holds, that $0<R^{-}<R<R^{+}$, that the right hand side is $F \in L^{2}(\Omega)$ and that the final time $0<T<\infty$ is given and bounded.

\subsection{Main result}

Definition 5.1. We define as a weak solution of the system $(2.5)-(2.7)$, any couple of functions $(\psi, \Phi)$ such that

- the following properties of regularity are satisfied

$$
\nabla \Phi, \nabla \psi \in L^{\infty}\left(0, T ; L^{2}(\Omega)\right) \quad \text { and } \quad \Delta \Phi, \Delta \psi \in L^{2}\left(0, T ; L^{2}(\Omega)\right) ;
$$

- the system $(2.5)$ holds in $\mathcal{D}^{\prime}((0, T) \times \Omega)$;

- the homogeneous Dirichlet boundary conditions (2.7) are satisfied in $\mathcal{D}^{\prime}(\partial \Omega)$

$$
\Phi=\psi=\frac{\partial \Phi}{\partial n}=0 \text { on } \partial \Omega .
$$

Theorem 5.2. Assume the initial data is $\left(\psi_{0}, \Phi_{0}\right) \in\left(H_{0}^{1}(\Omega) \cap H^{2}(\Omega)\right) \times H_{0}^{2}(\Omega)$. There exists a weak solution $(\Phi, \psi)$ of $(2.5)-(2.7)$ in the sense of Definition 5.1. Moreover, the weak solution satisfies $(\Phi, \psi)_{t=0}=\left(\Phi_{0}, \psi_{0}\right)$.

This result is proved using sequence of approximate solutions constructed in (5.2), with a convenient splitting in time. The time step is denoted as $\Delta t>0$.

\subsection{Proof of Theorem 5.2}

The sequence is defined incrementally for all $k$ such that $k \Delta t \leq T$. It writes in strong form as

$$
\left\{\begin{array}{l}
\frac{\psi_{\Delta t}^{k+1}-\psi_{\Delta t}^{k}}{R \Delta t}=\frac{1}{\rho R^{2}}\left[\psi_{\Delta t}^{k}, \Phi_{\Delta t}^{k+1}\right]+\frac{\eta}{R} \Delta^{\star} \psi_{\Delta t}^{k+1}-\frac{\eta F}{R}, \\
\frac{\omega_{\Delta t}^{k+1}-\omega_{\Delta t}^{k}}{\rho R \Delta t}=\left[\frac{\omega_{\Delta t}^{k}}{\rho^{2} R^{2}}, \Phi_{\Delta t}^{k+1}\right]+\left[\psi_{\Delta t}^{k}, \frac{1}{\rho R^{2}} \Delta^{\star} \psi_{\Delta t}^{k+1}\right]+\frac{\nu}{\rho R} \Delta_{\rho} \omega_{\Delta t}^{k+1},
\end{array}\right.
$$

with the initialization

$$
\psi_{\Delta t}^{0}=\psi_{0}, \quad \Phi_{\Delta t}^{0}=\Phi_{0} \quad \text { and } \omega_{\Delta t}^{0}=\omega_{0}=\Delta_{\rho} \Phi_{0} .
$$

It is convenient to consider the weak form which defines this sequence with a convenient use of the boundary conditions. Let us multiply the first equation of (5.2) by a smooth test function $-\Delta^{\star} \widetilde{\psi}$ with

$$
\widetilde{\psi} \in H_{0}^{1}(\Omega) \cap H^{2}(\Omega) .
$$


We obtain

$$
\begin{gathered}
\int_{\Omega} \frac{\nabla \psi_{\Delta t}^{k+1} \cdot \nabla \widetilde{\psi}}{R}+\eta \Delta t \int_{\Omega} \frac{\Delta^{\star} \psi_{\Delta t}^{k+1} \Delta^{\star} \widetilde{\psi}}{R}+\Delta t \int_{\Omega} \frac{1}{\rho R^{2}}\left[\psi_{\Delta t}^{k}, \Phi_{\Delta t}^{k+1}\right] \Delta^{\star} \widetilde{\psi} \\
=\int_{\Omega} \frac{\nabla \psi_{\Delta t}^{k} \cdot \nabla \widetilde{\psi}}{R}+\Delta t \int_{\Omega} \frac{\eta F}{R} \Delta^{\star} \widetilde{\psi} \quad \forall \widetilde{\psi} \in H_{0}^{1}(\Omega) \cap H^{2}(\Omega) .
\end{gathered}
$$

We perform the same integration by parts for the second equation: we multiply by a test function $-\widetilde{\Phi}$ with

$$
\widetilde{\Phi} \in H_{0}^{2}(\Omega) .
$$

We obtain

$$
\begin{gathered}
\int_{\Omega} \frac{\nabla \Phi_{\Delta t}^{k+1} \cdot \nabla \widetilde{\Phi}}{\rho R}+\nu \Delta t \int_{\Omega} \frac{\Delta_{\rho} \psi_{\Delta t}^{k+1} \Delta_{\rho} \widetilde{\psi}}{R}-\Delta t \int_{\Omega} \frac{1}{\rho R^{2}}\left[\psi_{\Delta t}^{k}, \widetilde{\Phi}\right] \Delta^{\star} \psi_{\Delta t}^{k+1} \\
+\Delta t \int_{\Omega}\left[\widetilde{\Phi}, \Phi_{\Delta t}^{k+1}\right] \frac{\omega_{\Delta t}^{k}}{\rho^{2} R^{2}}=\int_{\Omega} \frac{\nabla \Phi_{\Delta t}^{k} \cdot \nabla \widetilde{\Phi}}{\rho R} \quad \forall \widetilde{\Phi} \in H_{0}^{2}(\Omega) .
\end{gathered}
$$

The unknowns of the system (5.3)-(5.4) are $\psi_{\Delta t}^{k+1}$ and $\Phi_{\Delta t}^{k+1}$. One recompute $\omega_{\Delta t}^{k+1}$ for the next time step using $\omega_{\Delta t}^{k+1}=\Delta_{\rho} \Phi_{\Delta t}^{k+1}$. The next proposition guarantees that the iterative procedure is well defined.

Proposition 5.3. Assume that the initial conditions are

$$
\left(\psi_{0}, \Phi_{0}\right) \in\left(H_{0}^{1}(\Omega) \cap H^{2}(\Omega)\right) \times H_{0}^{2}(\Omega) .
$$

The approximate sequence defined by the weak formulation (5.3)-(5.4) is well defined at each time step. Moreover there exists a constant $C$ such that for all $k \geq 0$

$$
\left\|\psi_{\Delta t}^{k}\right\|_{H_{0}^{1}(\Omega) \cap H^{2}(\Omega)}+\left\|\Phi_{\Delta t}^{k}\right\|_{H_{0}^{2}(\Omega)} \leq C .
$$

Proof. The proof by recurrence on $k$ is very classical. One checks that the bilinear forms of the variational problem (5.3)-(5.4) are bicontinuous and coercive in $\left(H_{0}^{1}(\Omega) \cap H^{2}(\Omega)\right) \times H_{0}^{2}(\Omega)$.

The important part of the proof concerns the bilinear forms

$$
\begin{aligned}
& (\widehat{\psi}, \widehat{\Phi} ; \widetilde{\psi}, \widetilde{\Phi}) \mapsto \int_{\Omega} \frac{1}{\rho R^{2}}\left[\psi_{\Delta t}^{k}, \widehat{\Phi}\right] \Delta^{\star} \widetilde{\psi} \\
& (\widehat{\psi}, \widehat{\Phi} ; \widetilde{\psi}, \widetilde{\Phi}) \mapsto \int_{\Omega} \frac{1}{\rho R^{2}}\left[\psi_{\Delta t}^{k}, \widetilde{\Phi}\right] \Delta^{\star} \widehat{\psi}
\end{aligned}
$$

and

$$
(\widehat{\psi}, \widehat{\Phi} ; \widetilde{\psi}, \widetilde{\Phi}) \mapsto \int_{\Omega}[\widetilde{\Phi}, \widehat{\Phi}] \frac{\omega_{\Delta t}^{k}}{\rho^{2} R^{2}}
$$

Indeed it is sufficient to use the embedding

$$
H_{0}^{1}(\Omega) \cap H^{2}(\Omega) \subset W^{1,4}(\Omega) \text { and } H_{0}^{2}(\Omega) \subset W^{1,4}(\Omega)
$$

to show these bilinear forms are bicontinuous in $\left(H_{0}^{1}(\Omega) \cap H^{2}(\Omega)\right) \times H_{0}^{2}(\Omega)$. The coercivity is evident: formally it is sufficient to take the test function $\widetilde{\psi}=\psi_{\Delta t}^{k+1}$ in (5.3) and the test function $\widetilde{\Phi}=\Phi_{\Delta t}^{k+1}$ in (5.4) and to add the two right hand sides (5.3)-(5.4); the contributions (5.5)-(5.7) vanish: therefore the result is coercive in $\left(H_{0}^{1}(\Omega) \cap H^{2}(\Omega)\right) \times H_{0}^{2}(\Omega)$. On the other hand the linear right hand side has the required continuity. Therefore the variational solution $\left(\psi_{\Delta t}^{k+1}, \Phi_{\Delta t}^{k+1}\right)$ exists and is unique by the Lax-Milgram theorem. Finally the Sobolev embedding in dimension two $H^{1}(\Omega) \subset L^{p}(\Omega)$ for all $1<p<\infty$ guarantees that

$$
H^{2}(\Omega) \subset W^{1,4}(\Omega) .
$$

Therefore $\left(\psi_{\Delta t}^{k}, \omega_{\Delta t}^{k}\right) \in W^{1,4}(\Omega) \times L^{4}(\Omega)$ which is sufficient to go to the next iteration. The proof is ended. 
It is convenient to define a continuous in time approximation by

$$
\begin{aligned}
& \psi_{\Delta t}(t)=\psi_{\Delta t}^{k}+\frac{t-k \Delta t}{\Delta t}\left(\psi_{\Delta t}^{k+1}-\psi_{\Delta t}^{k}\right), \\
& \Phi_{\Delta t}(t)=\Phi_{\Delta t}^{k}+\frac{t-k \Delta t}{\Delta t}\left(\Phi_{\Delta t}^{k+1}-\Phi_{\Delta t}^{k}\right)
\end{aligned}
$$

and

$$
\omega_{\Delta t}(t)=\omega_{\Delta t}^{k}+\frac{t-k \Delta t}{\Delta t}\left(\omega_{\Delta t}^{k+1}-\omega_{\Delta t}^{k}\right)=\Delta_{\rho} \Phi_{\Delta t}(t),
$$

for $k \Delta t \leq t \leq(k+1) \Delta t$. These functions are continuous in time (in appropriate functional spaces).

For technical purposes we also define piecewise constant in time functions

$$
\begin{aligned}
& \overline{\psi_{\Delta t}}(t)=\psi_{\Delta t}^{k} \text { for } k \Delta t<t<(k+1) \Delta t, \\
& \overline{\Phi_{\Delta t}}(t)=\Phi_{\Delta t}^{k} \text { for } k \Delta t<t<(k+1) \Delta t
\end{aligned}
$$

and

$$
\overline{\omega_{\Delta t}}(t)=\omega_{\Delta t}=\Delta_{\rho} \bar{\Phi}_{\Delta t}^{n}(t) \text { for } k \Delta t<t<(k+1) \Delta t .
$$

With these notations (5.3) rewrites naturally as

$$
\frac{1}{R} \partial_{t} \psi_{\Delta t}(t)=\frac{1}{\rho R^{2}}\left[\overline{\psi_{\Delta t}}(t), \overline{\Phi_{\Delta t}}(t+\Delta t)\right]+\frac{\eta}{R} \Delta^{\star} \overline{\psi_{\Delta t}}(t+\Delta t)-\frac{\eta}{R} F, \quad k \Delta t<t<(k+1) \Delta t,
$$

in the sense of distributions in space. Similarly (5.4) may naturally be rewritten as

$\frac{1}{\rho R} \partial_{t} \omega_{\Delta t}(t)=\left[\frac{\overline{\omega_{\Delta t}}(t)}{\rho^{2} R^{2}}, \overline{\Phi_{\Delta t}}(t+\Delta t)\right]+\left[\overline{\psi_{\Delta t}}(t), \frac{1}{\rho R^{2}} \Delta^{\star} \overline{\psi_{\Delta t}}(t+\Delta t)\right]+\frac{\nu}{\rho R} \Delta_{\rho} \overline{\omega_{\Delta t}}(t+\Delta t), \quad k \Delta t<t<(k+1) \Delta t$,

also in the sense of distributions in space. The equation for $\partial_{t} \Phi_{\Delta t}$ may also be rewritten but it is less direct since it involves the inverse of $\Delta_{\rho}$. This is why we prefer to use (5.11) for the simplicity of mathematical analysis.

\subsubsection{A priori estimates}

Following the proof of Proposition 4.3, we notice that the boundary conditions (2.7) make all boundary terms, appearing in the integrations by parts, vanish. Then we obtain the following energy identity which is a generalization of the continuous energy identity (4.1).

Lemma 5.4. One has the inequality for all $k \in \mathbb{N}$,

$$
\begin{aligned}
\int_{\Omega}\left(\frac{\left|\nabla \psi_{\Delta t}^{k+1}\right|^{2}}{2 R}\right. & \left.+\frac{\left|\nabla \Phi_{\Delta t}^{k+1}\right|^{2}}{2 \rho R}\right) \mathrm{d} x+\eta \Delta t \sum_{0 \leq l \leq k} \int_{\Omega} \frac{\left|\Delta^{\star} \psi_{\Delta t}^{l+1}\right|^{2}}{R} \mathrm{~d} x+\nu \Delta t \sum_{0 \leq l \leq k} \int_{\Omega} \frac{\left|\Delta_{\rho} \Phi_{\Delta t}^{l+1}\right|^{2}}{\rho R} \mathrm{~d} x \\
& \leq \int_{\Omega}\left(\frac{\left|\nabla \psi_{0}\right|^{2}}{2 R}+\frac{\left|\nabla \Phi_{0}\right|^{2}}{2 \rho R}\right) \mathrm{d} x+\eta \Delta t \sum_{0 \leq l \leq k} \int_{\Omega} \frac{F \Delta^{\star} \psi_{\Delta t}^{l+1}}{R} \mathrm{~d} x .
\end{aligned}
$$

Proof. We take $\widetilde{\psi}=\psi_{\Delta t}^{k+1}$ in (5.3) and to take $\widetilde{\Phi}=\Phi_{\Delta t}^{k+1}$ in (5.4). After summation and simplification we obtain

$$
\begin{aligned}
& \int_{\Omega}\left(\frac{\left|\nabla \psi_{\Delta t}^{k+1}\right|^{2}}{R}+\frac{\left|\nabla \Phi_{\Delta t}^{k+1}\right|^{2}}{\rho R}\right) \mathrm{d} x+\eta \Delta t \int_{\Omega} \frac{\left|\Delta^{\star} \psi_{\Delta t}^{k+1}\right|^{2}}{R} \mathrm{~d} x+\nu \Delta t \int_{\Omega} \frac{\left|\Delta_{\rho} \Phi_{\Delta t}^{k+1}\right|^{2}}{\rho R} \mathrm{~d} x \\
& =\int_{\Omega}\left(\frac{\nabla \psi_{\Delta t}^{k} \cdot \nabla \psi_{\Delta t}^{k+1}}{R}+\frac{\nabla \Phi_{\Delta t}^{k} \cdot \nabla \Phi_{\Delta t}^{k+1}}{\rho R}\right) \mathrm{d} x+\eta \Delta t \int_{\Omega} \frac{F \Delta^{\star} \psi_{\Delta t}^{k+1}}{R} \mathrm{~d} t \\
& \leq \int_{\Omega}\left(\frac{\left|\nabla \psi_{\Delta t}^{k+1}\right|^{2}}{2 R}+\frac{\left|\nabla \Phi_{\Delta t}^{k+1}\right|^{2}}{2 \rho R}\right) \mathrm{d} x+\int_{\Omega}\left(\frac{\left|\nabla \psi_{\Delta t}^{k}\right|^{2}}{2 R}+\frac{\left|\nabla \Phi_{\Delta t}^{k}\right|^{2}}{2 \rho R}\right) \mathrm{d} x+\eta \Delta t \int_{\Omega} \frac{F \Delta^{\star} \psi_{\Delta t}^{k+1}}{R} \mathrm{~d} t .
\end{aligned}
$$


Therefore

$$
\begin{gathered}
\int_{\Omega}\left(\frac{\left|\nabla \psi_{\Delta t}^{k+1}\right|^{2}}{2 R}+\frac{\left|\nabla \Phi_{\Delta t}^{k+1}\right|^{2}}{2 \rho R}\right) \mathrm{d} x+\eta \Delta t \int_{\Omega} \frac{\left|\Delta^{\star} \psi_{\Delta t}^{k+1}\right|^{2}}{R} \mathrm{~d} x+\nu \Delta t \int_{\Omega} \frac{\left|\Delta_{\rho} \Phi_{\Delta t}^{k+1}\right|^{2}}{\rho R} \mathrm{~d} x \\
\leq \int_{\Omega}\left(\frac{\left|\nabla \psi_{\Delta t}^{k}\right|^{2}}{2 R}+\frac{\left|\nabla \Phi_{\Delta t}^{k}\right|^{2}}{2 \rho R}\right) \mathrm{d} x+\eta \Delta t \int_{\Omega} \frac{F \Delta^{\star} \psi_{\Delta t}^{k+1}}{R} \mathrm{~d} t
\end{gathered}
$$

which yields the claim after summation with respect to $k$.

Lemma 5.5. Any sequence $\psi_{\Delta t}$ and $\Phi_{\Delta t}(5.8)-(5.9)$ satisfies

$$
\psi_{\Delta t}, \Phi_{\Delta t} \text { bounded in } L^{\infty}\left(0, T ; H_{0}^{1}(\Omega)\right) \cap L^{2}\left(0, T ; H^{2}(\Omega)\right)
$$

independently of $\Delta t$.

Moreover, for all $\xi \in] 0,1[$, independently of $\Delta t$, we have

$$
\left.\nabla \psi_{\Delta t}, \nabla \Phi_{\Delta t} \text { bounded in } \mathcal{K}_{\xi}=L^{\frac{2}{1-\xi}}\left(0, T ; L^{s}(\Omega)\right), \text { for all } s \in\right] 2, \frac{2}{\xi}[.
$$

Therefore, there exists functions $(\psi, \Phi)$ such that

$$
\begin{aligned}
& \psi_{\Delta t} \rightarrow \psi \text { and } \Phi_{\Delta t} \rightarrow \Phi \text { weakly in } L^{\infty}\left(0, T ; H_{0}^{1}(\Omega)\right) \cap L^{2}\left(0, T ; H^{2}(\Omega)\right), \\
& \left.\nabla \psi_{\Delta t} \rightarrow \nabla \psi \text { and } \nabla \Phi_{\Delta t} \rightarrow \nabla \Phi \text { weakly in } \mathcal{K}_{\xi}, \text { for all } \xi \in\right] 0,1[ \\
& \Delta^{\star} \psi_{\Delta t} \rightarrow \Delta^{\star} \psi \text { and } \omega_{\Delta t} \rightarrow \omega \text { weakly in } L^{2}\left(0, T ; L^{2}(\Omega)\right) .
\end{aligned}
$$

Proof. Let $k$ be any integer such that $k \Delta t \leq T$. The bounds in $\psi_{\Delta t}^{k+1}, \Phi_{\Delta t}^{k+1} \in H_{0}^{1}(\Omega)$ uniformely with respect to $k$ are straightforward from (5.12) with a standard Gronwall lemma, since $\rho$ and $R$ are bounded from above and from below and since the Poincaré inequality is guaranteed by the homogeneous Dirichlet boundary conditions. The function $\psi_{\Delta t}$ is an average between $\psi_{\Delta t}^{k}$ and $\psi_{\Delta t}^{k+1}$

$$
\psi_{\Delta t}(t)=\left(1-\frac{t-k \Delta t}{\Delta t}\right) \psi_{\Delta t}^{k}+\frac{t-k \Delta t}{\Delta t} \psi_{\Delta t}^{k+1}, \quad k \Delta t<t<(k+1) \Delta t .
$$

So $\psi_{\Delta t}, \Phi_{\Delta t} \in L^{\infty}\left(0, T ; H_{0}^{1}(\Omega)\right)$.

The bounds $\overline{\psi_{\Delta t}}(\cdot+\Delta t), \overline{\Phi_{\Delta t}}(\cdot+\Delta t) \in L^{2}\left(0, T ; H^{2}(\Omega)\right)$ are also straightforward from (5.12).

Since $\psi_{0} \in H^{2}(\Omega)$ by hypothesis and $\overline{\psi_{\Delta t}}(\cdot+\Delta t) \in L^{2}\left(0, T ; H^{2}(\Omega)\right)$ due to (5.12), then $\psi_{\Delta t}$ is uniformly bounded in $L^{2}\left(0, T ; H^{2}(\Omega)\right)$. The situation is the same for $\Phi_{\Delta t}$. It proves (5.13).

The bounds (5.14) are obtained by interpolation. Indeed, the bounds (5.13), together with the Sobolev embedding in dimension two $H^{1}(\Omega) \subset L^{p}(\Omega)$ for all $1<p<+\infty$, imply that $\nabla \psi_{\Delta t}$ and $\nabla \Phi_{\Delta t}$ are bounded in $L^{\infty}\left(0, T ; L^{2}(\Omega)\right)$ and $L^{2}\left(0, T ; L^{p}(\Omega)\right)$ for all $1<p<+\infty$. The conclusion (5.14) comes from the embedding

$$
L^{\infty}\left(0, T ; L^{2}(\Omega)\right) \cap L^{2}\left(0, T ; L^{p}(\Omega)\right) \subset L^{r}\left(0, T ; L^{s}(\Omega)\right)
$$

for all $r, s$ such that

$$
\frac{1}{r}=\frac{1-\xi}{2}, \quad \frac{1}{s}=\frac{\xi}{2}+\frac{1-\xi}{p}
$$

with any $\xi \in] 0,1[$ and any $1<p<+\infty$. 


\subsubsection{Compactness properties of $\left(\psi_{\Delta t}, \Phi_{\Delta t}\right)$}

In order to have some compactness properties, we want to use the following theorem.

Theorem 5.6 (in [27], Thm. 5.1, p. 58). Let $B$ be a Banach space, and $B_{0}$ and $B_{1}$ be two reflexive Banach spaces. Assume $B_{0} \subset B$ with compact injection, $B \subset B_{1}$ with continuous injection.

Fix $T<+\infty, 1<p_{0}<+\infty, 1<p_{1}<+\infty$. Then the injection

$$
\left\{v \in L^{p_{0}}\left(0, T ; B_{0}\right) ; \partial_{t} v \in L^{p_{1}}\left(0, T ; B_{1}\right)\right\} \subset L^{p_{0}}(0, T ; B)
$$

is compact.

To be able to use this theorem in our context, we have to find convenient bounds on the time derivative of the magnetic and velocity potentials.

For the magnetic potential one can use for convenience the equation in strong form (5.10) for almost all $t \geq 0$

$$
\partial_{t} \psi_{\Delta t}(t)=\underbrace{\frac{1}{\rho R}\left[\overline{\psi_{\Delta t}}(t), \overline{\Phi_{\Delta t}}(t+\Delta t)\right]}_{S_{1}}+\underbrace{\eta \Delta^{\star} \overline{\psi_{\Delta t}}(t+\Delta t)}_{S_{2}}-\eta F .
$$

As remarked in the previous proof, we immediately know that the second term $S_{2}$ belongs to $L^{2}\left(0, T ; L^{2}(\Omega)\right)$. The first term $S_{1}(\cdot)=\frac{1}{\rho R} \nabla \overline{\psi_{\Delta t}}(\cdot) \cdot \nabla^{\perp} \overline{\Phi_{\Delta t}}(\cdot+\Delta t)$ belongs to $L^{\infty}\left(0, T ; L^{2}(\Omega)\right) \cap L^{2}\left(0, T ; H_{0}^{1}(\Omega)\right)$. Since $H_{0}^{1}(\Omega) \subset L^{p}(\Omega)$ for all $1<p<+\infty$, then $S_{1} \in L^{2}\left(0, T ; L^{s}(\Omega)\right)$, for all $1<s<2$.

Through (5.15) and thinking that $\rho$ and $R$ are bounded from below, we can conclude that $\partial_{t} \psi_{\Delta t}$ is bounded in $L^{2}\left(0, T ; L^{s}(\Omega)\right)$, for all $1<s<2$.

Then, using Theorem 5.6 with $p_{0}=p_{1}=2$ and $B_{0}=H^{2}(\Omega) \subset B=W^{1, p}(\Omega) \subset B_{1}=L^{s}(\Omega)$, we can insure that

$$
\psi_{\Delta t} \rightarrow \psi \text { in } L^{2}\left(0, T ; W^{1, p}(\Omega)\right) \text {, for all } 1<p<+\infty .
$$

For convenience the boundary and initial conditions will be addressed later.

For the velocity potential one can start from the equation which holds in the sense of distribution with respect to the space variable and for almost all $t>0$

$$
\partial_{t} \omega_{\Delta t}(t)=\underbrace{\rho R\left[\frac{\overline{\omega_{\Delta t}}(t)}{\rho^{2} R^{2}}, \overline{\Phi_{\Delta t}}(t+\Delta t)\right]}_{T_{1}}+\underbrace{\rho R\left[\overline{\psi_{\Delta t}}(t), \frac{1}{\rho R^{2}} \Delta^{\star} \overline{\psi_{\Delta t}}(t+\Delta t)\right]}_{T_{2}}+\underbrace{\nu \Delta_{\rho} \overline{\omega_{\Delta t}}(t+\Delta t)}_{T_{3}} .
$$

Let's deal with the bounds on $T_{1}, T_{2}$ and $T_{3}$. Notice that we will sometimes forget $\rho$ and $R$ coefficients which are bounded from above and from below. The Poisson brackets reads also

$$
[a, b]=\operatorname{div}\left(a \nabla^{\perp} b\right),
$$

where $\nabla^{\perp}=\left(-\partial_{y}, \partial_{x}\right)$.

We can say that $\overline{\omega_{\Delta t}}(t) \nabla^{\perp} \overline{\Phi_{\Delta t}}(t+\Delta t)=\Delta_{\rho} \overline{\Phi_{\Delta t}}(t) \nabla^{\perp} \overline{\Phi_{\Delta t}}(t+\Delta t)$ is the product of one function in $L^{2}\left(0, T ; L^{2}(\Omega)\right)$ and the other one in $\mathcal{K}_{\xi}$ : the result belongs to $L^{\frac{2}{2-\xi}}\left(0, T ; L^{s}(\Omega)\right)$, for all $1<s<\frac{2}{1+\xi}$, and for any $\xi \in] 0,1[$.

Since $\rho R$ is smooth and bounded from below, then there exists $r>1$ such that $T_{1}$ and $T_{2}$ are bounded in $L^{\frac{2}{2-\xi}}\left(0, T ; W^{-1, s}(\Omega)\right)+L^{\frac{2}{2-\xi}}\left(0, T ; L^{r}(\Omega)\right)$, for all $1<s<\frac{2}{1+\xi}$. But, in 2-dimension, $L^{r}(\Omega) \subset H^{-1}(\Omega)$, for all $r>1$, so we can summarize as follows: $T_{1}, T_{2}$ are all bounded in $L^{\frac{2}{2-\xi}}\left(0, T ; W^{-1, s}(\Omega)\right)$, for all $1<s<\frac{2}{1+\xi}$.

On the other hand, since $\overline{\omega_{\Delta t}}$ is bounded in $L^{2}\left(0, T ; L^{2}(\Omega)\right)$, we conclude that $T_{3}$ is bounded in $L^{2}\left(0, T ; H^{-2}(\Omega)\right)$. 
As a consequence, by equation (5.17), the time derivative $\partial_{t} \overline{\omega_{\Delta t}}$ is bounded in $L^{2}\left(0, T ; H^{-2}(\Omega)\right)$. Therefore the time derivative $\partial_{t} \overline{\Phi_{\Delta t}}$ is bounded in $L^{2}\left(0, T ; L^{2}(\Omega)\right)^{3}$. It allows us to use Theorem 5.6 to conclude

$$
\Phi_{\Delta t} \rightarrow \Phi \text { in } L^{2}\left(0, T ; W^{1, p}(\Omega)\right) \text {, for all } 1<p<+\infty .
$$

One also has that

$$
\omega_{\Delta t} \rightarrow \omega \text { in } L^{2}\left(0, T ; W^{-1, p}(\Omega)\right), \text { for all } 1<p<+\infty .
$$

\subsubsection{Boundary conditions and initial condition}

Since the limit belongs to the same space one gets that $\psi$, which is the limit of $\psi_{\Delta t}$, is such that $\psi \in$ $L^{2}\left(0, T ; H_{0}^{1}(\Omega)\right)$. Therefore the homogeneous condition $\psi=0$ holds in $\mathcal{D}^{\prime}(\partial \Omega)$. Similarily, $\Phi \in L^{2}\left(0, T ; H_{0}^{2}(\Omega)\right)$. That's why the other boundary condition $\Phi=\frac{\partial \Phi}{\partial n}=0$ also holds in the sense of distributions.

We also notice that both $\psi_{\Delta t}$ and $\Phi_{\Delta t}$ are uniformly bounded in $W^{1, s}(] 0, T[\times \Omega)$ for small $s>1$. Therefore the trace of these functions is well defined at $t=0$. It yield the fact that the limit also belongs to the same space, and that the initial condition is true after passing to the limit $\Delta t \rightarrow 0$. That is

$$
\psi(0)=\psi_{0} \text { and } \Phi(0)=\Phi_{0} .
$$

\subsubsection{Compactness of $\left(\overline{\psi_{\Delta t}}, \overline{\Phi_{\Delta t}}\right)$}

The function $\overline{\psi_{\Delta t}}$ is an approximation constant by step of the function $\psi_{\Delta t}$. For $k \Delta t<t<(k+1) \Delta t$, one has $\overline{\psi_{\Delta t}}(t)-\psi_{\Delta t}(t)=(t-k \Delta t) \partial_{t} \psi_{\Delta t}(t)$ by definition of these functions. Therefore $\left\|\overline{\psi_{\Delta t}}(t)-\psi_{\Delta t}(t)\right\|_{L^{s}(\Omega)} \leq$ $\Delta t\left\|\partial_{t} \psi_{\Delta t}(t)\right\|_{L^{s}(\Omega)}$. Since $\partial_{t} \psi_{\Delta t}$ is bounded in $L^{2}\left(0, T ; L^{s}(\Omega)\right), 1<s<2$, uniformly with respect to $\Delta t>0$ it yields

$$
\overline{\psi_{\Delta t}}-\psi_{\Delta t} \rightarrow 0 \text { in } L^{2}\left(0, T ; L^{s}(\Omega)\right), \quad 1<s<2 .
$$

On the other hand $\overline{\psi_{\Delta t}}$ and $\psi_{\Delta t}$ are bounded in $L^{2}\left(0, T ; W^{1, p^{\prime}}(\Omega)\right)$ for all $1<p^{\prime}<p$. By interpolation between $L^{2}\left(0, T ; W^{1, p^{\prime}}(\Omega)\right)$ and $L^{2}\left(0, T ; L^{s}(\Omega)\right)$ we obtain that

$$
\overline{\psi_{\Delta t}}-\psi_{\Delta t} \rightarrow 0 \text { in } L^{2}\left(0, T ; W^{1, p}(\Omega)\right), \quad \text { for all } 1<p<\infty .
$$

Therefore (at least a subsequence)

$$
\overline{\psi_{\Delta t}} \rightarrow \psi \text { in } L^{2}\left(0, T ; W^{1, p}(\Omega)\right) \text {, for all } 1<p<+\infty .
$$

That is the stepwise function also tends to the same limit.

We have similarly (at least a subsequence)

$$
\overline{\Phi_{\Delta t}} \rightarrow \Phi \text { in } L^{2}\left(0, T ; W^{1, p}(\Omega)\right) \text {, for all } 1<p<+\infty
$$

and

$$
\overline{\omega_{\Delta t}} \rightarrow \omega \text { in } L^{2}\left(0, T ; W^{-1, p}(\Omega)\right), \text { for all } 1<p<+\infty
$$

\footnotetext{
${ }^{3} \mathrm{~A}$ more direct way to the result, once it is known, is to remark that (5.4) can be rewritten for $k \Delta t<t<(k+1) \Delta t$ and for all $\widetilde{\Phi} \in H_{0}^{2}(\Omega)$, as

$$
\int_{\Omega} \frac{1}{\rho R} \partial_{t} \Phi_{\Delta t}(t) \Delta^{\star} \widetilde{\Phi}=\nu \Delta t \int_{\Omega} \frac{\Delta_{\rho} \psi_{\Delta t}^{k+1} \Delta_{\rho} \widetilde{\psi}}{R}-\Delta t \int_{\Omega} \frac{1}{\rho R^{2}}\left[\psi_{\Delta t}^{k}, \widetilde{\Phi}\right] \Delta^{\star} \psi_{\Delta t}^{k+1}+\Delta t \int_{\Omega}\left[\widetilde{\Phi}, \Phi_{\Delta t}^{k+1}\right] \frac{\omega_{\Delta t}^{k}}{\rho^{2} R^{2}}
$$

The supremum of the right hand side over all $\widetilde{\Phi} \in H^{2}(\Omega)$, together with a convenient use of the various a priori bounds, yields the control in $L^{2}(\Omega)$ of the time derivative $\partial_{t} \Phi_{\Delta t}(t)$ over the time step $k \Delta t<t<(k+1) \Delta t$. After integration in time it yields $(5.20)$.
} 


\subsubsection{Convergences}

We start from equations (5.10)-(5.11). The concern is the quadratic terms $S_{1}, T_{1}, T_{2}$ in (5.15)-(5.17). Since the Poisson brackets can be rewritten as in (5.18), we only need to get the convergence in the sense of distributions for the terms of " $a \nabla^{\perp} b$ "-type.

- In order to pass to the limit in equation (5.10), we just have to deal with the term $\overline{\psi_{\Delta t}}(\cdot) \nabla^{\perp} \overline{\Phi_{\Delta t}}(\cdot+\Delta t)$. We know that $\overline{\psi_{\Delta t}}(\cdot)$ strongly converges to $\psi$ in $L^{2}\left(0, T ; L^{p}(\Omega)\right)$, for all $1<p<+\infty$ and that $\nabla^{\perp} \overline{\Phi_{\Delta t}}(\cdot+\Delta t)$ weakly converges to $\nabla^{\perp} \Phi L^{2}\left(0, T ; L^{p}(\Omega)\right)$, for all $1<p<+\infty$ (see (5.14)). This is enough to conclude the convergence for $S_{1}$

$$
\overline{\psi_{\Delta t}}(\cdot) \nabla^{\perp} \overline{\Phi_{\Delta t}}(\cdot+\Delta t) \rightarrow \psi \nabla^{\perp} \Phi \quad \text { in } \mathcal{D}^{\prime}((0, T) \times \Omega) ;
$$

- for equation (5.11), three terms are concerned.

Since $\nabla^{\perp} \overline{\Phi_{\Delta t}}(\cdot+\Delta t)$ strongly converges to $\nabla^{\perp} \Phi$ in $L^{2}\left(0, T ; L^{p}(\Omega)\right)$, for all $1<p<+\infty$ and since $\overline{\omega_{\Delta t}}(\cdot)$ weakly converges to $\omega$ in $L^{2}\left(0, T ; L^{2}(\Omega)\right)$, we get the expected convergence for $T_{1}$ :

$$
\overline{\omega_{\Delta t}}(\cdot) \nabla^{\perp} \overline{\Phi_{\Delta t}}(\cdot+\Delta t) \rightarrow \omega \nabla^{\perp} \Phi \text { in } \mathcal{D}^{\prime}((0, T) \times \Omega) .
$$

Analogous arguments can be expressed for the convergence of $T_{2}$ :

$$
\Delta^{\star} \overline{\psi_{\Delta t}}(\cdot+\Delta t) \nabla^{\perp} \overline{\psi_{\Delta t}}(\cdot) \rightarrow \Delta^{\star} \psi \nabla^{\perp} \psi \text { in } \mathcal{D}^{\prime}((0, T) \times \Omega) .
$$

Once again an alternative way to pass to the limit is to start directly from (5.19).

\section{Stability of Stationary SOLUtions}

In this section we study the stability of stationary solutions. The ultimate goal in the context of magnetic plasma is to determine what are the stationary solutions which are also stable solutions. It is possible to relax the assumption of stationarity, in this case the questions is to determine quasi-stationary and stable solutions. In what follows we focus on spectral stability of a special family of stationary solutions which correspond to eigenvectors of the Grad-Shafranov operator. What is remarkable is that the stability estimates do not blow up as $\eta, \nu \rightarrow 0$ : inequality (6.13) is independent of $\eta, \nu$, and inequality (6.23)-(6.25) improve as $\eta$ becomes smaller.

We consider strong solutions of the problem (2.5)-(2.7). The initial conditions are denoted

$$
\psi_{0}=\psi(t=0), \quad \omega_{0}=\omega(t=0) .
$$

We will use assume in this section that $\nu>0, \eta>0$, that (2.6) holds, and that $0<R^{-}<R<R^{+}$.

\subsection{Stationary solutions}

In our context, a stationary solution is such that the velocity is zero, which turns into $\Phi_{0}=\omega_{0}=0$. In this case

$$
-\Delta^{\star} \psi_{0}=J_{c} \text { and } \omega_{0}=0 .
$$

Plugging in (2.5) one gets the relation

$$
\left[\psi_{0}, \frac{1}{\rho R^{2}} \Delta^{\star} \psi_{0}\right]=0
$$

Any $\psi_{0}$ such that $\frac{1}{\rho R^{2}} \Delta^{\star} \psi_{0}=f\left(\psi_{0}\right)$ for a given function $f$ satisfies this condition. In this work we assume a spectral dependence that is

$$
-\frac{1}{\rho R^{2}} \Delta^{\star} \psi_{0}=\lambda \psi_{0}
$$

Equivalently $\psi_{0}$ is solution to

$$
\begin{cases}-\partial_{R}\left(\frac{1}{R} \partial_{R} \psi_{0}\right)-\partial_{Z}\left(\frac{1}{R} \partial_{Z} \psi_{0}\right)=\lambda \rho R \psi_{0}, & x \in \Omega, \\ \psi_{0}=0, & x \in \partial \Omega .\end{cases}
$$


This problem admits a symmetric and positive weak formulation in $H_{0}^{1}(\Omega)$, see [1]. We deduce that there exists a complete family $\left(u_{i}, \lambda_{i}\right)_{i \geq 0}$ of real eigenvectors $u_{i} \in H_{0}^{1}(\Omega)$ and real eigenvalues $\lambda_{i} \in \mathbb{R}$ such that

$$
\begin{cases}-\partial_{R}\left(\frac{1}{R} \partial_{R} u_{i}\right)-\partial_{Z}\left(\frac{1}{R} \partial_{Z} u_{i}\right)=\lambda_{i} \rho R u_{i}, & x \in \Omega, \\ u_{i}=0, & x \in \partial \Omega,\end{cases}
$$

with the ordering

$$
0<\lambda_{0}<\lambda_{1} \leq \lambda_{2} \leq \cdots
$$

The spectral gap is positive [1]

$$
\mu=\lambda_{1}-\lambda_{0}>0 .
$$

The eigenvectors are orthonormal for the weighted $L^{2}$ scalar product and for the weighted $H_{0}^{1}$ scalar product

$$
\int_{\Omega} \rho R u_{i} u_{j}=\delta_{i j} \text { and } \int_{\Omega} \frac{1}{R} \nabla u_{i} \cdot \nabla u_{j}=\lambda_{i} \delta_{i j} .
$$

Definition 6.1. All initial data

$$
\left(\psi_{0}, \omega_{0}\right)=\gamma\left(u_{i}, 0\right), \quad \forall i
$$

are stationary for the source term $J_{c}=\Delta^{\star} u_{i}$. We may call them spectral initial data.

Remark 6.2. The eigenvalues and eigenvectors are continuous with respect to the coefficient of the problem which is $\rho$. Equivalently the eigenvalues and eigenvectors are continuous with respect to the function

$$
w=\rho R^{2} .
$$

With (6.4), bounds of various quantities are obtained uniformly with respect to the density profile.

\subsection{Stability in the case $i=0$}

We show in this section that the first eigenfunction has strong stability properties. As a preliminary remark we stress that the generalization of (4.1) yields

$$
\frac{\mathrm{d}}{\mathrm{dt}} \int_{\Omega} \frac{|\nabla \psi|^{2}}{2 R}+\frac{|\nabla \Phi|^{2}}{2 \rho R}+\eta \int_{\Omega} \frac{\left(\Delta^{\star} \psi\right)^{2}}{R}+\nu \int_{\Omega} \frac{\omega^{2}}{\rho R}=\eta \int_{\Omega} \frac{1}{R} F \Delta^{\star} \psi
$$

from which we can deduce from the Cauchy-Schwarz inequality that

$$
\frac{\mathrm{d}}{\mathrm{dt}} \int_{\Omega} \frac{|\nabla \psi|^{2}}{2 R}+\frac{|\nabla \Phi|^{2}}{2 \rho R} \leq \eta \int_{\Omega} \frac{1}{2 R} F^{2}
$$

It implies that $\psi$ and $\Phi$ remain bounded in the $H_{0}^{1}$ norm

$$
\|\psi(t)\|_{H_{0}^{1}(\Omega)}^{2}+\|\Phi(t)\|_{H_{0}^{1}(\Omega)}^{2} \leq C\left(\left\|\psi_{0}\right\|_{H_{0}^{1}(\Omega)}^{2}+t\|F\|_{L^{2}(\Omega)}^{2}\right) .
$$

This inequality does not imply nor that $\psi(t)$ remains close to its initial condition $\psi_{0}$, neither that $\Phi(t)$ remains close to zero. Our goal is here to obtain some inequalities that will explain precisely that $\psi(t)$ remains close to its initial condition $\psi_{0}$, and that $\Phi(t)$ remains close to zero. It will establish the stability of the corresponding initial data.

Let us define the coefficients $\beta_{n}$ of expansion of $\psi$ over the eigenvector basis

$$
\psi(t)=\sum_{n \geq 0} \beta_{n}(t) u_{n}, \quad \beta_{n}(t)=\int_{\Omega} \rho R \psi(t) u_{n} .
$$


We set $\gamma=\beta_{0}(0)$. We study the differences

$$
\bar{\psi}=\psi-\gamma u_{0} \quad \text { and } \quad \bar{\omega}=\omega-0=\omega .
$$

We will make use of the expansion

$$
\bar{\psi}(t)=\sum_{n \geq 0} \alpha_{n}(t) u_{n}, \quad \alpha_{n}(t)=\int_{\Omega} \rho R \bar{\psi}(t) u_{n}, \quad \alpha_{0}(0)=0 .
$$

Similarly we define $\bar{\Phi}=\Phi-\Phi_{0}=\Phi$. We also assume that $J_{c}=\gamma \Delta^{\star} u_{0}$.

Proposition 6.3. One has the identity

$$
\frac{\mathrm{d}}{\mathrm{dt}} \int_{\Omega}\left(\frac{|\nabla \bar{\psi}|^{2}}{2 R}-\frac{\lambda_{0} \rho R \bar{\psi}^{2}}{2}+\frac{|\nabla \bar{\Phi}|^{2}}{2 \rho R}\right)=-\eta \mathcal{I}(\bar{\psi})-\nu \int_{\Omega} \frac{\bar{\omega}^{2}}{\rho R}
$$

where we have defined

$$
\mathcal{I}(\bar{\psi})=\int_{\Omega}\left(\frac{\left(\Delta^{\star} \bar{\psi}\right)^{2}}{R}+\lambda_{0} \rho R \bar{\psi} \Delta^{\star} \bar{\psi}\right) .
$$

Proof. Simple algebra shows that

$$
\left\{\begin{aligned}
\partial_{t} \bar{\psi}= & \frac{1}{\rho R}\left[\gamma u_{0}, \bar{\Phi}\right] \\
& +\frac{1}{\rho R}[\bar{\psi}, \bar{\Phi}]+\eta \Delta^{\star} \bar{\psi} \\
\partial_{t} \bar{\omega}= & \rho R\left[\gamma u_{0}, \frac{1}{\rho R^{2}} \Delta^{\star} \bar{\psi}\right]+\rho R\left[\bar{\psi}, \frac{1}{\rho R^{2}} \Delta^{\star} \gamma u_{0}\right] \\
& +\rho R\left(\frac{1}{(\rho R)^{2}}[\bar{\omega}, \bar{\Phi}]+\left[\frac{1}{(\rho R)^{2}}, \bar{\Phi}\right] \bar{\omega}\right)+\rho R\left[\bar{\psi}, \frac{1}{\rho R^{2}} \Delta^{\star} \bar{\psi}\right]+\nu \Delta_{\rho} \bar{\omega}
\end{aligned}\right.
$$

The source term vanishes by construction. The right hand sides are the sum of a linear term with respect to $\bar{\psi}$ and $\bar{\omega}$ (this term is written just after the sign $=$ ) and of a quadratic term (written on the next line). Concerning the quadratic terms, the structure is identical to the structure of the system (2.5). Multiplying $\partial_{t} \bar{\psi}$ by $-\frac{1}{R} \Delta^{\star} \bar{\psi}$ and $\partial_{t} \bar{\omega}$ by $-\frac{1}{\rho R} \bar{\Phi}$ and integrating by parts in the domain, the following energy relation can be deduced

$$
\begin{aligned}
\frac{\mathrm{d}}{\mathrm{dt}} \int_{\Omega} \frac{|\nabla \bar{\psi}|^{2}}{2 R}+\frac{|\nabla \bar{\Phi}|^{2}}{2 \rho R}= & -\eta \int_{\Omega} \frac{\left(\Delta^{\star} \bar{\psi}\right)^{2}}{R}-\nu \int_{\Omega} \frac{\bar{\omega}^{2}}{\rho R}-\int_{\Omega}\left(\frac{1}{\rho R}\left[\gamma u_{0}, \bar{\Phi}\right]\right)\left(\frac{1}{R} \Delta^{\star} \bar{\psi}\right) \\
& -\int_{\Omega}\left(\rho R\left[\gamma u_{0}, \frac{1}{\rho R^{2}} \Delta^{\star} \bar{\psi}\right]+\rho R\left[\bar{\psi}, \frac{1}{\rho R^{2}} \Delta^{\star} \gamma u_{0}\right]\right)\left(\frac{1}{\rho R} \bar{\Phi}\right) \\
= & -\eta \int_{\Omega} \frac{\left(\Delta^{\star} \bar{\psi}\right)^{2}}{R}-\nu \int_{\Omega} \frac{\bar{\omega}^{2}}{\rho R}-\int_{\Omega}\left[\gamma u_{0}, \bar{\Phi}\right] \frac{1}{\rho R^{2}} \Delta^{\star} \bar{\psi}-\int_{\Omega}\left[\gamma u_{0}, \frac{1}{\rho R^{2}} \Delta^{\star} \bar{\psi}\right] \bar{\Phi} \\
& -\int_{\Omega}\left[\bar{\psi}, \frac{1}{\rho R^{2}} \Delta^{\star} \gamma u_{0}\right] \bar{\Phi} \\
= & -\eta \int_{\Omega} \frac{\left(\Delta^{\star} \bar{\psi}\right)^{2}}{R}+\lambda_{0} \int_{\Omega}\left[\bar{\psi}, \gamma u_{0}\right] \bar{\Phi}-\nu \int_{\Omega} \frac{\bar{\omega}^{2}}{\rho R} \\
= & -\eta \int_{\Omega} \frac{\left(\Delta^{\star} \bar{\psi}\right)^{2}}{R}+\lambda_{0} \int_{\Omega}\left[\gamma u_{0}, \bar{\Phi}\right] \bar{\psi}-\nu \int_{\Omega} \frac{\bar{\omega}^{2}}{\rho R} .
\end{aligned}
$$


Next we eliminate $\left[\gamma u_{0}, \bar{\Phi}\right]$ with the first equation of the system rewritten as

$$
\left[\gamma u_{0}, \bar{\Phi}\right]=\rho R \partial_{t} \bar{\psi}-[\bar{\psi}, \bar{\Phi}]-\eta \rho R \Delta^{\star} \bar{\psi}
$$

so that

$$
\begin{aligned}
\int_{\Omega}\left[\gamma u_{0}, \bar{\Phi}\right] \bar{\psi} & =\int_{\Omega} \rho R \partial_{t} \frac{\bar{\psi}^{2}}{2}-\int_{\Omega}\left[\frac{\bar{\psi}^{2}}{2}, \bar{\Phi}\right]-\eta \int_{\Omega} \rho R\left(\Delta^{\star} \bar{\psi}\right) \bar{\psi} \\
& =\int_{\Omega} \rho R \partial_{t} \frac{\bar{\psi}^{2}}{2}-\eta \int_{\Omega} \rho R\left(\Delta^{\star} \bar{\psi}\right) \bar{\psi} .
\end{aligned}
$$

The remaining step consists in showing that the left hand side is a non negative quadratic form, and that the right hand side is controlled. To do so, let us define

$$
\widehat{\psi}(t)=\bar{\psi}(t)-\alpha_{0}(t) u_{0}=\sum_{n \geq 1} \alpha_{n}(t) u_{n} .
$$

Proposition 6.4. The left hand side of (6.8) controls the $H_{0}^{1}$ norm of $\widehat{\psi}$ and $\bar{\Phi}$. There exists a constant $C>0$ such that

$$
\mu\|\widehat{\psi}\|_{H_{0}^{1}(\Omega)}^{2}+\|\bar{\Phi}\|_{H_{0}^{1}(\Omega)}^{2} \leq C \int_{\Omega}\left(\frac{|\nabla \bar{\psi}|^{2}}{R}-\lambda_{0} \rho R \bar{\psi}^{2}+\frac{|\nabla \bar{\Phi}|^{2}}{\rho R}\right),
$$

where $\mu$ is the spectral gap (6.1).

Proof. It is sufficient to remark that

$$
\int_{\Omega} \rho R \bar{\psi}^{2}=\sum_{n \geq 0} \alpha_{n}^{2} \quad \text { and } \quad \int_{\Omega} \frac{|\nabla \bar{\psi}|^{2}}{R}=\sum_{n \geq 0} \lambda_{n} \alpha_{n}^{2} .
$$

Therefore

$$
\int_{\Omega}\left(\frac{|\nabla \bar{\psi}|^{2}}{R}-\lambda_{0} \rho R \bar{\psi}^{2}\right)=\sum_{n \geq 1}\left(\lambda_{n}-\lambda_{0}\right) \alpha_{n}^{2} \geq \mu \sum_{n \geq 1} \alpha_{n}^{2}
$$

controls the $H_{0}^{1}$ norm of $\widehat{\psi}$.

Let us define for convenience

$$
\|\widehat{\psi}, \bar{\Phi}\| \|^{2}=\int_{\Omega}\left(\frac{|\nabla \bar{\psi}|^{2}}{2 R}-\lambda_{0} \rho R \bar{\psi}^{2}+\frac{|\nabla \bar{\Phi}|^{2}}{2 \rho R}\right)
$$

which is an equivalent $H_{0}^{1}(\Omega)$ norm of $\widehat{\psi}$ and $\bar{\Phi}$ due to the inequality (6.11).

Proposition 6.5. Assume that the function $w$ defined in (6.4) is constant. Then

$$
\|\widehat{\psi}(t), \bar{\Phi}(t)\| \leq\|\widehat{\psi}(0), \bar{\Phi}(0)\|, \quad \forall \nu, \quad \eta>0 .
$$

Proof. The hypothesis $\rho R=\frac{w}{R}$ has a major consequence. Indeed the contribution to be analyzed in the right hand side of $(6.8)$ is $\mathcal{I}(\bar{\psi})$. 
One has

$$
\begin{aligned}
\mathcal{I}(\bar{\psi}) & =\sum_{n, m \geq 0} \alpha_{n} \alpha_{m} \int_{\Omega} \lambda_{n} \lambda_{m} \frac{\rho^{2} R^{4}}{R} u_{n} u_{m}-\lambda_{0} \lambda_{m} \rho^{2} R^{3} u_{n} u_{m} \\
& =w \sum_{n, m \geq 0} \alpha_{n} \alpha_{m} \int_{\Omega}\left(\lambda_{n} \lambda_{m}-\lambda_{0} \lambda_{m}\right) \rho R u_{n} u_{m} .
\end{aligned}
$$

Because of the orthogonality relations (6.2), we obtain

$$
\mathcal{I}(\bar{\psi})=w \sum_{n} \alpha_{n}^{2}\left(\lambda_{n}^{2}-\lambda_{0} \lambda_{n}\right) .
$$

Therefore $\mathcal{I}(\bar{\psi}) \geq 0$ unconditionally and the claim is proved.

Next we do not consider anymore that $w$ is a constant. The method of analysis consists nevertheless in comparing $\mathcal{I}(\bar{\psi})$ with a functional that can be decomposed as in (6.14). But we first establish technical results.

Proposition 6.6. Let $T>0$. The coefficient $\alpha_{0}(t)$ satisfies the estimate

$$
\left|\alpha_{0}(t)\right| \leq C \int_{0}^{t}\|\widehat{\psi}(s)\|_{H_{0}^{1}(\Omega)} \mathrm{d} s
$$

for some constant $C>0$ and for all $t \leq T$.

Proof. Using the first equation of (6.10) one obtains

$$
\alpha_{0}^{\prime}(t)=\frac{\mathrm{d}}{\mathrm{dt}} \int_{\Omega} \rho R \bar{\psi}(t) u_{0}=\int_{\Omega}\left[\gamma u_{0}, \bar{\Phi}\right] u_{0}+\int_{\Omega}[\bar{\psi}, \bar{\Phi}] u_{0}+\eta \int_{\Omega} \rho R \Delta^{\star} \bar{\psi} u_{0} .
$$

The first integral vanishes: $\int_{\Omega}\left[\gamma u_{0}, \bar{\Phi}\right] u_{0}=\gamma \int_{\Omega}\left[u_{0}, u_{0}\right] \bar{\Phi}=0$. The second integral is

$$
\int_{\Omega}[\bar{\psi}, \bar{\Phi}] u_{0}=\int_{\Omega}\left[\bar{\psi}-\alpha_{0}(t) u_{0}, \bar{\Phi}\right] u_{0}=\int_{\Omega}[\widehat{\psi}, \bar{\Phi}] u_{0} .
$$

Using the energy identity (6.6) it is evident that $\bar{\Phi}$ is bounded in $H_{0}^{1}(\Omega)$. Therefore

$$
\left|\int_{\Omega}[\bar{\psi}, \bar{\Phi}] u_{0}\right| \leq C\|\widehat{\psi}\|_{H_{0}^{1}(\Omega)} .
$$

The third integral is

$$
\begin{aligned}
\int_{\Omega} \rho R \Delta^{\star} \bar{\psi} u_{0} & =\int_{\Omega} \rho R \Delta^{\star} \widehat{\psi} u_{0}+\alpha_{0}(t) \int_{\Omega} \rho R\left(\Delta^{\star} u_{0}\right) u_{0} \\
& =\int_{\Omega} \rho R \Delta^{\star} \widehat{\psi} u_{0}-\alpha_{0}(t) \lambda_{0} \int_{\Omega} \rho^{2} R^{3} u_{0}^{2} .
\end{aligned}
$$

One also has after one integration by parts

$$
\left|\int_{\Omega} \rho R \Delta^{\star} \widehat{\psi} u_{0}\right| \leq C\|\widehat{\psi}\|_{H_{0}^{1}(\Omega)} .
$$

Therefore one has the Gronwall type relation $\alpha_{0}^{\prime}(t)+\sigma \alpha_{0}(t)=q(t)$ with $\sigma=\lambda_{0} \int_{\Omega} \rho^{2} R^{3} u_{0}^{2}>0$ and $|q(t)| \leq$ $C\|\widehat{\psi}(t)\|_{H_{0}^{1}(\Omega)}$ for some universal constant $C>0$. So

$$
\alpha_{0}(t)=\int_{0}^{t} \mathrm{e}^{-\sigma(t-s)} q(s) \mathrm{d} s
$$

since $\alpha_{0}(0)=0$ due to (6.7). This proves the claim. 
Lemma 6.7 (Gronwall lemma). Let $t \mapsto f(t)$ be a smooth non negative function such that

$$
f(t) \leq A+B \int_{0}^{t} f(s) \mathrm{d} s+C \int_{0}^{t} \int_{0}^{s} f(r) \mathrm{d} r, \quad B \geq 0, A, C>0 .
$$

One has the inequalities:

$$
f(t) \leq \begin{cases}A \mathrm{e}^{\alpha C t} & \text { if } B \neq 0 \\ A \cosh (\alpha C t) & \text { if } B=0\end{cases}
$$

where $\alpha$ denotes the positive solution of $C \alpha^{2}-B \alpha-1=0$, say, $\alpha=\frac{B+\sqrt{B^{2}+4 C}}{2 C}$.

Proof. Set

$$
u(t)=\int_{0}^{t} \int_{0}^{s} f(r) \mathrm{d} r \text { and } v=\alpha u^{\prime}+u,
$$

where the parameter $\alpha$ is defined as the positive solution of $C \alpha^{2}-B \alpha-1=0$.

Then one successively gets

$$
\begin{aligned}
v^{\prime}(t)=\alpha u^{\prime \prime}(t)+u^{\prime}(t)=\alpha f(t)+u^{\prime}(t) & \leq \alpha\left(A+B u^{\prime}(t)+C u(t)\right)+u^{\prime}(t) \\
& \leq \alpha A+(\alpha B+1) u^{\prime}(t)+\alpha C u(t) \\
& \leq \alpha A+\alpha C\left(\alpha u^{\prime}(t)+u(t)\right) \\
& \leq \alpha A+\alpha C v(t)
\end{aligned}
$$

From $v^{\prime}(t)-\alpha C v(t) \leq \alpha A$, a classical Gronwall lemma then gives

$$
v(t) \leq \frac{A}{C}\left(\mathrm{e}^{\alpha C t}-1\right)
$$

First case: $B \neq 0$.

By hypothesis, one has $f(t) \leq A+B u^{\prime}(t)+C u(t)$, and since $B \leq B+\frac{1}{\alpha}=\alpha C$ one gets

$$
f(t) \leq A+C\left(\alpha u^{\prime}(t)+u(t)\right)=A+C v(t) .
$$

Putting it together with (6.17) the final inequality $f(t) \leq A \mathrm{e}^{\alpha C t}$ is obtained.

Second case: $B=0$.

The relation defining $\alpha$ now writes $\alpha^{2} C=1$.

Let us come back to (6.17):

$$
\alpha u^{\prime}(t)+u(t)=v(t) \leq \frac{A}{C}\left(\mathrm{e}^{\alpha C t}-1\right) .
$$

Once more, a Gronwall lemma between 0 and $t$ shows that

$$
u(t) \leq \frac{A}{C}\left(\frac{\mathrm{e}^{\alpha C t}+\mathrm{e}^{-\alpha C t}}{2}-1\right) .
$$

Since $f(t) \leq A+C u(t)$, the second inequality $f(t) \leq A \cosh (\alpha C t)$ is proved.

Let us define for convenience

$$
w_{-}=\min _{(R, Z) \in \Omega} w(R, Z)>0 .
$$


Proposition 6.8. There exists a second order polynomial

$$
x \mapsto p_{t}(x)=-C_{1} \beta\left|\alpha_{0}(t)\right| x+C_{2} \mu x^{2}-C_{3} \beta x^{2}
$$

where $\mu$ is the spectral gap, $\beta=\left\|w-w_{-}\right\|_{W^{1, \infty}(\Omega)}$, and the three constants $C_{1}, C_{2}$ and $C_{3}$ are positive, such that

$$
\mathcal{I}(\bar{\psi}(t)) \geq p_{t}\left(\|\widehat{\psi}\|_{H_{0}^{1}(\Omega)}\right)
$$

Proof. We first remark that $\bar{\psi}(t)=\widehat{\psi}(t)+\alpha_{0}(t) u_{0}$, so that

$$
\begin{aligned}
\mathcal{I}(\bar{\psi}) & =\mathcal{I}(\widehat{\psi})+\alpha_{0}(t)^{2} \mathcal{I}\left(u_{0}\right)+\alpha_{0}(t) \int_{\Omega} \frac{2 \Delta^{\star} u_{0} \Delta^{\star} \widehat{\psi}}{R}+\lambda_{0} \rho R\left(u_{0} \Delta^{\star} \widehat{\psi}+\widehat{\psi} \Delta^{\star} u_{0}\right) \\
& =\mathcal{I}(\widehat{\psi})+\alpha_{0}(t) \int_{\Omega} \lambda_{0} \rho R\left(-u_{0} \Delta^{\star} \widehat{\psi}+\widehat{\psi} \Delta^{\star} u_{0}\right)
\end{aligned}
$$

after simplifications.

- The integral term is changed for convenience into

$$
\begin{aligned}
\int_{\Omega} \lambda_{0} \frac{w}{R}\left(-u_{0} \Delta^{\star} \widehat{\psi}+\widehat{\psi} \Delta^{\star} u_{0}\right) & =\int_{\Omega} \lambda_{0} \frac{w-w_{-}}{R}\left(-u_{0} \Delta^{\star} \widehat{\psi}+\widehat{\psi} \Delta^{\star} u_{0}\right)+w_{-} \int_{\Omega} \frac{\lambda_{0}}{R}\left(-u_{0} \Delta^{\star} \widehat{\psi}+\widehat{\psi} \Delta^{\star} u_{0}\right) \\
& =\int_{\Omega} \lambda_{0} \frac{w-w_{-}}{R}\left(-u_{0} \Delta^{\star} \widehat{\psi}+\widehat{\psi} \Delta^{\star} u_{0}\right) .
\end{aligned}
$$

We also define

$$
\beta=\left\|w-w_{-}\right\|_{W^{1, \infty}(\Omega)} .
$$

Then

One gets

$$
\left|\int_{\Omega} \lambda_{0} \frac{w-w_{-}}{R}\left(-u_{0} \Delta^{\star} \widehat{\psi}+\widehat{\psi} \Delta^{\star} u_{0}\right)\right| \leq C_{1} \beta\|\widehat{\psi}\|_{H_{0}^{1}(\Omega)} .
$$

$$
\left|\alpha_{0}(t) \int_{\Omega} \lambda_{0} \rho R\left(-u_{0} \Delta^{\star} \widehat{\psi}+\widehat{\psi} \Delta^{\star} u_{0}\right)\right| \leq C_{1}\left|\alpha_{0}(t)\right| \beta\|\widehat{\psi}\|_{H_{0}^{1}(\Omega)} .
$$

- Let us define the integral

Due to the orthogonality relations (6.2)

$$
\mathcal{J}(\widehat{\psi})=\int_{\Omega} \frac{\left(\Delta^{\star} \widehat{\psi}\right)^{2}}{\rho R^{3}}+\frac{\lambda_{0}}{R} \widehat{\psi} \Delta^{\star} \widehat{\psi} .
$$

$$
\mathcal{J}(\widehat{\psi})=\sum_{n \geq 0} \alpha_{n}^{2}\left(\lambda_{n}^{2}-\lambda_{0} \lambda_{n}\right) \geq 0
$$

holds without condition. Notice also that one has

$$
\mathcal{J}(\widehat{\psi}) \geq\left(\lambda_{1}-\lambda_{0}\right) \sum_{n \geq 1} \lambda_{n} \alpha_{n}^{2} \geq C_{2}\left(\lambda_{1}-\lambda_{0}\right)\|\widehat{\psi}\|_{H_{0}^{1}(\Omega)}^{2} .
$$

- Next,

$$
\begin{aligned}
\mathcal{I}(\widehat{\psi}) & =\int_{\Omega} w \frac{\left(\Delta^{\star} \widehat{\psi}\right)^{2}}{\rho R^{3}}+w \frac{\lambda_{0}}{R} \widehat{\psi} \Delta^{\star} \widehat{\psi} \\
& =\int_{\Omega}\left(\left(w-w_{-}\right) \frac{\left(\Delta^{\star} \widehat{\psi}\right)^{2}}{\rho R^{3}}+\left(w-w_{-}\right) \frac{\lambda_{0}}{R} \widehat{\psi} \Delta^{\star} \widehat{\psi}\right)+w_{-} \mathcal{J}(\widehat{\psi}) .
\end{aligned}
$$


Then

$$
\mathcal{I}(\widehat{\psi}) \geq w_{-} \mathcal{J}(\widehat{\psi})+\int_{\Omega}\left(w-w_{-}\right) \frac{\lambda_{0}}{R} \widehat{\psi} \Delta^{\star} \widehat{\psi} .
$$

Thanks to (6.21) one has the lower bound

$$
\mathcal{I}(\widehat{\psi}) \geq C_{2}\left(\lambda_{1}-\lambda_{0}\right)\|\widehat{\psi}\|_{H^{1}(\Omega)}^{2}+\int_{\Omega}\left(w-w_{-}\right) \frac{\lambda_{0}}{R} \widehat{\psi} \Delta^{\star} \widehat{\psi}
$$

After one integration by parts, it comes

$$
\left|\int_{\Omega}\left(w-w_{-}\right) \frac{\lambda_{0}}{R} \widehat{\psi} \Delta^{\star} \widehat{\psi}\right| \leq C_{3}\left\|w-w_{-}\right\|_{W^{1, \infty}(\Omega)}\|\widehat{\psi}\|_{H^{1}(\Omega)}^{2} \quad\left(C_{3}>0\right) .
$$

Therefore one obtains

$$
\mathcal{I}(\bar{\psi}) \geq-C_{1}\left|\alpha_{0}(t)\right|\left\|w-w_{-}\right\|_{W^{1, \infty}(\Omega)}\|\widehat{\psi}\|_{H^{1}(\Omega)}+C_{2}\left(\lambda_{1}-\lambda_{0}\right)\|\widehat{\psi}\|_{H^{1}(\Omega)}^{2}-C_{3}\left\|w-w_{-}\right\|_{W^{1, \infty}(\Omega)}\|\widehat{\psi}\|_{H^{1}(\Omega)}^{2}
$$

or also $\mathcal{I}(\bar{\psi}) \geq p_{t}\left(\|\widehat{\psi}\|_{H_{0}^{1}(\Omega)}\right)$, and the proof is ended.

Theorem 6.9. Let $0<\eta \leq 1$. There exists a constant $L>0$ such that

$$
\forall t \leq T, \quad\|\widehat{\psi}(t), \bar{\Phi}(t)\| \mid \leq \mathrm{e}^{\sqrt{\eta} L t}\|\widehat{\psi}(0), \bar{\Phi}(0)\| \|
$$

More precisely, if the function $w$ has small variation in the sense

$$
\beta=\left\|w-w_{-}\right\|_{W^{1, \infty}(\Omega)}<c_{0}\left(\lambda_{1}-\lambda_{0}\right) \quad\left(c_{0}>0\right),
$$

then there exists a constant $L>0$ such that

$$
\forall t \leq T, \quad\||\widehat{\psi}(t), \bar{\Phi}(t)\||\leq \cosh (\sqrt{\eta} L t)\||| \widehat{\psi}(0), \bar{\Phi}(0)\| \| .
$$

Remark 6.10. This result shows that the growth of the perturbation is bounded by an exponential where the constant is small for small $\eta$. Moreover the initial growth is of second order for small $t$ provided $w$ has small variation. For example $\eta=10^{-4}$ in the simulations reported in [13] in the context of the Current Hole instability in Tokamaks. With this respect, it shows that small variation $w$ profiles display enhance stability behavior at initial time.

Proof.

(i) One can lower bound the negative part of $p_{t}(6.18)$ as

$$
p_{t}(x) \geq-M\left(\left|\alpha_{0}(t)\right|+x\right) x, \quad x \geq 0,
$$

where $M>0$ is a constant. Inserting in (6.8), (6.9)-(6.19), one obtains

$$
\frac{\mathrm{d}}{\mathrm{dt}}\||| \widehat{\psi}(t), \bar{\Phi}(t) \mid\|^{2} \leq \eta M\left(\left|\alpha_{0}(t)\right|+\|\widehat{\psi}(t)\|\right)\|\widehat{\psi}(t)\| .
$$

Due to (6.11) one can simplify

$$
\frac{\mathrm{d}}{\mathrm{dt}}\||\widehat{\psi}(t), \bar{\Phi}(t)|\| \leq \eta N\left(\left|\alpha_{0}(t)\right|+\||| \widehat{\psi}(t), \bar{\Phi}(t)|\||) .\right.
$$


Let us set $f(t)=\||\widehat{\psi}(t), \bar{\Phi}(t) \|| \mid$ for convenience. After integration one gets

$$
\begin{aligned}
f(t) & \leq f(0)+\eta N \int_{0}^{t} f(s) \mathrm{d} s+\eta N \int_{0}^{t}\left|\alpha_{0}(s)\right| \mathrm{d} s \\
& \leq f(0)+\eta N \int_{0}^{t} f(s) \mathrm{d} s+\eta P \int_{0}^{t} \int_{0}^{s} f(r) \mathrm{d} r \mathrm{~d} s
\end{aligned}
$$

for some constants $N$ and $P$. Lemma 6.7 applied to this inequality with $B=\eta N$ and $C=\eta P$ shows the claim (6.23). The constant $\alpha C$ defined in Lemma 6.7 is

$$
\alpha C=\frac{\eta N+\sqrt{\eta^{2} N^{2}+4 \eta P}}{2 \eta P} \eta P \leq \sqrt{\eta} L \quad \text { since } 0<\eta \leq 1
$$

(ii) for a small enough constant $c_{0}$, the dominant coefficient of $p_{t}(x)$ is positive: $C_{2} \mu-C_{3} \beta=C_{4}>0$.

Therefore

$$
p_{t}(x)=C_{4} x^{2}-C_{1} \beta\left|\alpha_{0}(t)\right| x \geq-M\left|\alpha_{0}(t)\right| x \quad \text { for } x \geq 0 .
$$

One obtains after simplifications

$$
f(t) \leq f(0)+\eta P \int_{0}^{t} \int_{0}^{s} f(r) \mathrm{d} r \mathrm{~d} s .
$$

The rest of the proof is evident, using Lemma 6.7.

\section{REFERENCES}

[1] G. Allaire, Numerical Analysis and Optimization: An Introduction to Mathematical Modelling and Numerical Simulation in Numerical Mathematics and Scientific Computation series. Oxford University Press (2007).

[2] D. Biskamp, Nonlinear Magnetohydrodynamics. Cambridge University Press (1992).

[3] J. Blum, Numerical simulation and optimal control in plasma physics, with application to Tokamaks. Series in Modern Applied Mathematics. Wiley/Gauthier-Villard (1989).

[4] J. Blum, Numerical identification of the plasma current density in a Tokamak fusion reactor: the determination of a non-linear source in an elliptic pde, invited conference, in Proceedings of PICOF02. Carthage, Tunisie (2002).

[5] J. Blum, T. Gallouet and J. Simon, Existence and control of plasma equilibirum in a Tokamak. SIAM J. Math. Anal. 17 (1986) 1158-1177.

[6] J. Blum, C. Boulbe and B. Faugeras, Real time reconstruction of plasma equilibrium in a Tokamak, International conference on burning plasma diagnostics. Villa Manoastero, Varenna (2007).

[7] H. Brezis and H. Berestycki, On a free boundary problem arising in plasma physics. Nonlinear Anal. 4 (1980) $415-436$.

[8] S. Briguglio, G. Wad, F. Zonca and C. Kar, Hybrid magnetohydrodynamic-gyrokinetic simulation of toroidal Alfven modes. Phys. Plasmas 2 (1995) 3711-3723.

[9] S. Briguglio, F. Zonca and C. Kar, Hybrid magnetohydrodynamic-particle simulation of linear and nonlinear evolution of Alfven modes in tokamaks. Phys. Plasmas 5 (1998) 3287-3301.

[10] L.A. Caffarelli and S. Salsa, A geometric approach to free boundary problems, Graduate Studies in Mathematics. AMS, Providence, RI 68 (2005).

[11] F. Chen, Introduction to plasma physics and controlled fusion. Springer, New York (1984).

[12] O. Czarny and G. Huysmans, MHD stability in X-point geometry: simulation of ELMs. Nucl. Fusion 47 (2007) 659-666.

[13] O. Czarny and G. Huysmans, Bézier surfaces and finite elements for MHD simulations. J. Comput. Phys. 227 (2008) $7423-7445$.

[14] E. Deriaz, B. Després, G. Faccanoni, K.P. Gostaf, L.-M. Imbert-Gérard, G. Sadaka and R. Sart, Magnetic equations with FreeFem++, The Grad-Shafranov equation and the Current Hole. ESAIM Proc. 32 (2011) 76-94.

[15] J.I. Diaz and J.F. Padial, On a free-boundary problem modeling the action of a limiter on a plasma. Discrete Contin. Dyn. Syst. Suppl. (2007) 313-322.

[16] J.I. Diaz and J.-M. Rakotoson, On a two-dimensional stationary free boundary problem arising in the confinement of a plasma in a Stellarator. C. R. Acad. Sci. Paris, Sér. I 317 (1993) 353-359.

[17] E. Feireisl, Dynamics of viscous compressible fluids. Oxford University Press (2004).

[18] J. Freidberg, Plasma physics and fusion energy. Cambridge (2007).

[19] A. Friedman, Variational principles and free-boundary problems. Wiley-interscience publication, Wiley, New York (1982). 
[20] T. Fujita, Tokamak equilibria with nearly zero central current: the current hole (review article). Nucl. Fusion 50 (2010).

[21] T. Fujita, T. Oikawa, T. Suzuki, S. Ide, Y. Sakamoto, Y. Koide, T. Hatae, O. Naito, A. Isayama, N. Hayashi and H. Shirai, Plasma equilibrium and confinement in a Tokamak with nearly zero central current density in JT-60U. Phys. Rev. Lett. 87 (2001) 245001-245005.

[22] J.F. Gerbeau, C. Le Bris and T. Lelièvre, Mathematical methods for the magnetohydrodynamics of liquid metals. Oxford University Press, USA (2006).

[23] G. Huysmans, T.C. Hender, N.C. Hawkes and X. Litaudon, MHD stability of advanced Tokamak scenarios with reversed central current: an explanation of the "Current Hole". Phys. Rev. Lett. 87 (2001) 245002-245006.

[24] G.T.A. Huysmans, S. Pamela, E. van der Plas and P. Ramet, Non-linear MHD simulations of edge localized modes (ELMs). Plasma Phys. Control. Fusion 51 (2009) 124012.

[25] B.B. Kadomtsev and O.P. Pogutse, Non linear helical perturbations of a plasma in a Tokamak. Sov. Phys.-JETP 38 (1974) $283-290$.

[26] S.-E. Kruger, C.C. Hegna and J.D. Callen, Generalized reduced magnetohydrodynamic equations. Phys. Plasmas 5 (1998) 4169-4183.

[27] J.-L. Lions, Quelques méthodes de résolution des problèmes aux limites non linéaires, Études Mathématiques. Dunod (1969).

[28] P.-L. Lions, Mathematical topics in fluid mechanics. Incompressible models, edited by Oxford Science Publication 1 (1996).

[29] P.-L. Lions, Mathematical topics in fluid mechanics. Compressible models, edited by Oxford Science Publication 2 (1998).

[30] H. Lütjens and J.-F. Luciani, The XTOR code for nonlinear 3D simulations of MHD instabilities in tokamak plasmas. J. Comput. Phys. 227 (2008) 6944-6966.

[31] H. Lütjens and J.-F. Luciani, XTOR-2F: A fully implicit NewtonKrylov solver applied to nonlinear 3D extended MHD in tokamaks. J. Comput. Phys. 229 (2010) 8130-8143.

[32] K. Miyamoto, Plasma physics and controlled nuclear fusion. Springer (2005).

[33] B. Nkonga, Private communication (2010).

[34] M.N. Rosenbluth, D.A. Monticello, H.R. Strauss and R.B. White, Dynamics of high $\beta$ plasmas. Phys. Fluids 19 (1976) 1987.

[35] R. Smaltz, Reduced, three-dimensional, nonlinear equations for high- $\beta$ plasmas including toroidal effects. Phys. Lett. A 82 (1981) $14-17$.

[36] H.R. Strauss, Nonlinear three-dimensional magnetohydrodynamics of noncircular Tokamaks. Phys. Fluids 19 (1976) $134-140$.

[37] H.R. Strauss, Dynamics of high $\beta$ plasmas. Phys. Fluids 20 (1977) 1354-1360.

[38] R. Temam, Remarks on a free boundary value problem arising in plasma physics. Commun. Partial Differ. Equ. 2 (1977) $563-585$.

[39] R. Temam, Navier-Stokes Equations, Theory and Numerical Analysis. North-Holland (1979).

[40] Z. Yoshida, S.M. Mahajan, S. Ohsaki, M. Iqbal and N. Shatashvili, Beltrami fields in plasmas: High-confinement mode boundary layers and high beta equilibria. Phys. Plasmas 8 (2001) 2125.

[41] Z. Yoshida et al., Potential Control and Flow Generation in a Toroidal Internal-Coil System - a New Approach to High-beta Equilibrium, in 20th IAEA Fusion Energy Conference. Online at http://www-naweb.iaea.org/napc/physics/fec/fec2004/papers/ic_p6-16.pdf (2004). 